\title{
Strong submeasures and applications to non-compact dynamical systems
}

\author{
TUYEN TRUNG TRUONG(D) \\ Department of Mathematics, University of Oslo, Blindern, 0851 Oslo, Norway \\ (e-mail: tuyentt@math.uio.no)
}

(Received 29 April 2020 and accepted in revised form 28 October 2020)

\begin{abstract}
A strong submeasure on a compact metric space $X$ is a sub-linear and bounded operator on the space of continuous functions on $X$. A strong submeasure is positive if it is non-decreasing. By the Hahn-Banach theorem, a positive strong submeasure is the supremum of a non-empty collection of measures whose masses are uniformly bounded from above. There are many natural examples of continuous maps of the form $f: U \rightarrow X$, where $X$ is a compact metric space and $U \subset X$ is an open-dense subset, where $f$ cannot extend to a reasonable function on $X$. We can mention cases such as transcendental maps of $\mathbb{C}$, meromorphic maps on compact complex varieties, or continuous self-maps $f: U \rightarrow U$ of a dense open subset $U \subset X$ where $X$ is a compact metric space. For the aforementioned mentioned the use of measures is not sufficient to establish the basic properties of ergodic theory, such as the existence of invariant measures or a reasonable definition of measure-theoretic entropy and topological entropy. In this paper we show that strong submeasures can be used to completely resolve the issue and establish these basic properties. In another paper we apply strong submeasures to the intersection of positive closed $(1,1)$ currents on compact Kähler manifolds.
\end{abstract}

Key words: invariant submeasures, open-dense defined maps, pullback and pushforward, strong submeasure, variational principle

2020 Mathematics Subject Classification: 37Axx (Primary); 32Hxx, 14E05 (Secondary)

\section{Introduction}

In dynamical systems and ergodic theory, measures play a crucial role. At least since Henri Poincaré, the first fundamental step for studying the dynamics of a continuous map $f: X \rightarrow X$ of a compact metric space $X$ is to construct invariant probability measures, that is, those measures $\mu$ for which $f_{*}(\mu)=\mu$, and in particular those with measure entropy equal to the topological entropy. A way to construct invariant measures 
is to start from a positive measure $\mu_{0}$, and then to consider any cluster points of the Cesaro average $(1 / n) \sum_{j=0}^{n}\left(f_{*}\right)^{j}\left(\mu_{0}\right)$. To this end, a crucial property is that we can push forward a probability measure by a continuous map and obtain another probability measure, and that this pushforward is linear on the space of measures. There is also the fundamental result $[14,15]$, called the variational principle, which relates measure entropies of invariant measures of a compact metric space and the topological entropy of the map. When we work with compact complex varieties, usually it is very difficult to construct dynamically interesting holomorphic maps $f: X \rightarrow X$, and one must be willing to deal with dominant meromorphic maps $f: X \rightarrow X$ in order to go forward. We can still define a notion of entropy for meromorphic maps, and when $X$ is Kähler the paper by Dinh and Sibony [11] relates this topological notion to geometrical/cohomological information (called dynamical degrees) of the map. However, so far only in very special cases are ideas from dynamics of diffeomorphisms of compact Riemann manifolds applicable to the study of meromorphic maps. For these special meromorphic maps, one can construct very special invariant measures $\mu$ with no mass on proper analytic sets, which can be pushed forward. (Here, we recall this pushforward for the reader's convenience. Let $\mu$ be a probability measure with no mass on proper analytic subsets of $X$. Then we define $f_{*}(\mu)$ as the extension by zero of the probability measure $\left(\left.f\right|_{X \backslash I(f)}\right)_{*}(\mu)$, where $I(f)$ is the indeterminacy set of $f$ and hence $f$ is holomorphic on $X \backslash I(f)$.) For the majority of meromorphic maps, however, there is no obvious such special invariant measure, and one faces difficulty in constructing interesting invariant measures by the Cesaro average as above, since the cluster points of these measures (even if the starting measure $\mu_{0}$ has no mass on proper analytic subsets) are not guaranteed to have no mass on proper analytic subsets. The problem is then that we really do not know how to push forward, in a reasonable way, a measure with support in the indeterminacy set $I(f)$ of $f$, and stay in the space of measures. For example, if $x_{0} \in I(f)$, then typically its image under the map $f$ will be of positive dimension, and there is no reasonable way to define the pushforward $f_{*}\left(\delta_{x_{0}}\right)$, of the Dirac measure at $x_{0}$, as a measure. Still, we can ask the following questions. Can we define instead the pushforward $f_{*}\left(\delta_{x_{0}}\right)$ as something else more general than a measure? More importantly, can we hope to obtain some analog of the fundamental results mentioned above for all meromorphic maps?

Besides the construction using Cesaro's average as above, when $X$ is Kähler, there is one other approach to constructing invariant measures, closely related to special properties of compact Kähler manifolds, by intersecting dynamically interesting special positive closed currents (so-called Green's currents). Intersection of positive closed currents, in particular those of bidgree $(1,1)$, is an interesting topic itself with many applications in complex geometry and complex dynamics, and has been intensively studied. The methods employed so far by most researchers in this topic are local in nature, and the resulting intersections are supposed to be positive measures. These local methods also usually provide answers which are not compatible with intersection in cohomology, and the latter is a consideration one needs to take into account in order for the definition to be meaningful. However, again here not much is known about what to do if the currents to intersect are too singular. For example, is there any meaning to assign to self-intersection of the current of integration on a line $L \subset \mathbb{P}^{2}$ ? For an even more interesting example, is there any meaning to assign 
to self-intersection of the current of integration on a curve $C$ in a compact Kähler surface whose self-intersection in cohomology is $\{C\} .\{C\}=-1$ ?

One key question is whether, for dynamics of dominant meromorphic maps, we should take more account of the indeterminacy set of the map as well as of its iterates (the latter point has so far not been very much pursued in the literature). Likewise, in considering intersection of positive closed currents, should we look more closely at the singular parts of the currents involved, instead of throwing them away (as in the local approaches mentioned above)?

All the maps mentioned above belong to a more general class of maps of the form: $f$ : $U \rightarrow X$, where $X$ is a compact metric space, $U \subset X$ is open-dense, and $f$ is a continuous map. Some natural examples of maps of this kind are transcendental maps $f: \mathbb{C} \rightarrow \mathbb{C}$, such as $f(z)=e^{z}$, where we choose $U=\mathbb{C}$ and $X=\mathbb{P}^{1}$ as the preferable compactification of $U$. A generalization of the latter maps are continuous maps $f: U \rightarrow U$, where $U$ is a non-compact metric space with a preferable compactification $X$. In this paper we give applications of strong submeasures, a classical but largely overlooked notion, to the dynamics of maps of these maps. Submeasures can also be applied to the question of intersection of positive closed currents on compact Kähler manifolds, which we will address in a separate paper.

To ease the presentation of the paper, we give a formal definition of the above class of maps.

Definition 1.1. Let $X$ and $Y$ be topological spaces. By an open-dense defined map between $X$ and $Y$ we mean that there exist an open-dense subset $U$ of $X$ and a map $f: U \rightarrow Y$. We emphasize that $f$ does not need to be defined on the whole of $X$. We denote such a map by $f: X \rightarrow Y$.

Moreover, if the map $f: U \rightarrow Y$ above is continuous, then we say that $f$ is a continuous open-dense defined map between $X$ and $Y$. In this case, we denote by OpenDom $(f)$ the largest such subset $U$, and by OpenIm( $f$ ) the image $f(\operatorname{OpenDom}(f))$. We also define the indeterminacy set of $f$ to be $I(f)=X \backslash \operatorname{OpenDom}(f)$.

We next define strong submeasures in detail. Let $X$ be a compact metric space. Denote by $\varphi \in C^{0}(X)$ the sup-norm $\|\varphi\|_{L^{\infty}}=\sup _{x \in X}|\varphi(x)|$. We recall that a functional $\mu: C^{0}(X) \rightarrow \mathbb{R}$ is sub-linear if $\mu\left(\varphi_{1}+\varphi_{2}\right) \leq \mu\left(\varphi_{1}\right)+\mu\left(\varphi_{2}\right)$ and $\mu(\lambda \varphi)=\lambda \mu(\varphi)$ for $\varphi_{1}, \varphi_{2}, \varphi \in C^{0}(X)$ and a non-negative constant $\lambda$. A strong submeasure is then simply a sub-linear functional $\mu: C^{0}(X) \rightarrow \mathbb{R}$ which is also bounded, that is, there is a constant $C>0$ so that for all $\varphi \in C^{0}(X)$ we have $|\mu(\varphi)| \leq C\|\varphi\|_{L^{\infty}}$. The least such constant $C$ is called the norm of $\mu$ and is denoted by $\|\mu\|$. A strong submeasure $\mu$ is positive if it is non-decreasing, that is, for all $\varphi_{1} \geq \varphi_{2}$ we have $\mu\left(\varphi_{1}\right) \geq \mu\left(\varphi_{2}\right)$. It is easy to check that a strong submeasure is Lipschitz continuous, $\left|\mu\left(\varphi_{1}\right)-\mu\left(\varphi_{2}\right)\right| \leq\|\mu\| \times\left\|\varphi_{1}-\varphi_{2}\right\|_{L^{\infty}}$, and convex, $\mu\left(t_{1} \varphi_{1}+t_{2} \varphi_{2}\right) \leq t_{1} \mu\left(\varphi_{1}\right)+t_{2} \mu\left(\varphi_{2}\right)$ for $t_{1}, t_{2} \geq 0$. We denote by $S M(X)$ the set of all strong submeasures on $X$, and $S M^{+}(X)$ the set of all positive strong submeasures on $X$.

By the Riesz representation theorem (see [18]), on $X$ a measure of bounded mass $\mu$ is the same as a linear operator $\mu: C^{0}(X) \rightarrow \mathbb{R}$, that is, $\mu\left(\lambda_{1} \varphi_{1}+\lambda_{2} \varphi_{2}\right)=\lambda_{1} \mu\left(\varphi_{1}\right)+\lambda_{2}\left(\varphi_{2}\right)$ 
for all $\varphi_{1}, \varphi_{2} \in C^{0}(X)$ and constants $\lambda_{1}, \lambda_{2}$. It is bounded $\left(|\mu(\varphi)| \leq C\|\varphi\|_{L^{\infty}}\right.$ for a constant $C$ independent of $\left.\varphi \in C^{0}(X)\right)$ and positive $\left(\mu(\varphi) \geq 0\right.$ whenever $\varphi \in C^{0}(X)$ is non-negative). Note that we can then choose $C=\mu(1)$ (the mass of the measure $\mu$ ) and by linearity the positivity is the same as having

$$
\mu\left(\varphi_{1}\right) \geq \mu\left(\varphi_{2}\right)
$$

for all $\varphi_{1}, \varphi_{2} \in C^{0}(X)$ satisfying $\varphi_{1} \geq \varphi_{2}$. For later reference, we denote by $M(X)$ the set of signed measures on $X$ and by $M^{+}(X)$ the set of positive measures on $X$. Note that $M(X) \subset S M(X)$ and $M^{+}(X) \subset S M^{+}(X)$.

The first main idea of this paper, to deal with the pushforward of a positive strong submeasure, is as follows. If $U \subset X$ is an open-dense set as above, then for any bounded continuous function $\psi: U \rightarrow \mathbb{R}$, there is a canonical way to extend it to a bounded upper-semicontinuous function $E(g): X \rightarrow \mathbb{R}$. Now, if $f: U \rightarrow Y$ is a continuous function (where $Y$ is an another compact metric space) and $\mu$ is a positive finite Borel measure on $X$, then we can define a pushforward $f_{*}(\mu)$ as a positive strong submeasure on $X$ in the following manner. If $\varphi: X \rightarrow \mathbb{R}$ is a continuous function, then $f^{*}(\varphi): U \rightarrow \mathbb{R}$ is a bounded continuous function, and hence we have the canonical extension $E\left(f^{*}(\varphi)\right)$ which is a bounded upper-semicontinuous function on $X$. Then we define

$$
\left(f_{*} \mu\right)(\varphi):=\mu\left(E\left(f^{*}(\varphi)\right)\right) .
$$

Details are given in $\$ 2$ where we show that the right-hand side of this definition gives rise to a strong positive submeasure and does not depend on the choice of $U$. Hence, this operator is well defined for continuous open-dense defined maps. The same idea can be applied to define more generally the pushforward of positive strong submeasures.

When $f: X \rightarrow X$ is a meromorphic map of a compact complex variety, we can choose $U$ to be any open-dense set of $X$ on which $f$ is a genuinely holomorphic function. In this case, because of Hironaka's resolution of singularities, one usually prefers to work with a desingularity of the graph of $f$. This is helpful in defining operations such as the pullback or pushforward of smooth closed forms resulting in currents. It will be shown that the definition given above for the pushforward of measures can also be made using these desingularities of graphs, and hence illustrates that our definition is reasonable.

When the map $f: U \rightarrow Y$ as above has dense image and is a covering map onto its image and of finite degree $d$ (for example, if this map is induced from a dominant meromorphic map between two compact complex spaces of the same dimension, and when $U$ is chosen appropriately), we can also define similarly the pullback of a positive strong submeasure. In Theorem 2.10 we prove some fundamental properties of these pushforward and pullback operators on positive strong submeasures. We extract here some interesting properties.

THEOREM 1.2. Let $X, Y$ be compact metric spaces, $U \subset X$ a dense open set, and $f$ : $X \rightarrow Y$ be a continuous open-dense defined map.

(i) If $\mu_{n} \in S M^{+}(X)$ weakly converges to $\mu$, and $v$ is a cluster point of $f_{*}\left(\mu_{n}\right)$, then $v \leq f_{*}(\mu)$. If $f$ is holomorphic, then $\lim _{n \rightarrow \infty} f_{*}\left(\mu_{n}\right)=f_{*}(\mu)$. 
(ii) For any positive strong submeasure $\mu$, we have $f_{*}(\mu)=\sup _{\chi \in \mathcal{G}(\mu)} f_{*}(\chi)$, where $\mathcal{G}(\mu)=\{\chi: \chi$ is a measure and $\chi \leq \mu\}$.

(iii) For positive strong submeasures $\mu_{1}, \mu_{2}$, we have $f_{*}\left(\mu_{1}+\mu_{2}\right) \geq f_{*}\left(\mu_{1}\right)+f_{*}\left(\mu_{2}\right)$.

In Example 2 in $\$ 2.2$ we show that strict inequality can occur in part (i) in general. It also shows that, in contrast to the case of a continuous map (see §4) part (ii) does not hold in general if we replace $\mathcal{G}(\mu)$ by a smaller set $\mathcal{G}$ (still satisfying $\mu=\sup _{\chi \in \mathcal{G}} \chi$ ). We also remark that several results in Theorem 2.10 (such as parts (1)-(3)) can easily be extended to meromorphic correspondences.

Before going further, let us calculate explicitly one simple but interesting example.

Example 1. Let $\pi: Y \rightarrow X$ be the blowup of $X$ at a point $p$, and $V \subset Y$ the exceptional divisor. Let $\delta_{p}$ be the Dirac measure at $p$. Then for any continuous function $\varphi$ on $Y$, we have

$$
\pi^{*}\left(\delta_{p}\right)(\varphi)=\max _{y \in V} \varphi(y) .
$$

Therefore, $\pi^{*}\left(\delta_{p}\right)$ is not a measure. In particular, if $A \subset Y$ is a closed set then $\pi^{*}\left(\delta_{p}\right)(A)=\inf _{\varphi \in C^{0}\left(X, \geq 1_{A}\right)} \pi^{*}\left(\delta_{p}\right)(\varphi)$ is $\delta_{p}(\pi(A \cap Y))$.

Proof of Example 1. By definition,

$$
\pi^{*}\left(\delta_{p}\right)(\varphi)=\inf _{\psi \in C^{0}\left(Y, \geq \pi_{*}(\varphi)\right)} \delta_{p}(\psi)=\inf _{\psi \in C^{0}\left(Y, \geq \pi_{*}(\varphi)\right)} \psi(p) .
$$

Since $\pi: Y \backslash V \rightarrow X \backslash\{p\}$ is an isomorphism, it is easy to check that $\pi_{*}(\varphi)(p)=$ $\max _{y \in V} \varphi(y)$. Therefore, for any $\psi \in C^{0}\left(Y, \geq \pi_{*}(\varphi)\right)$, we have $\psi(p) \geq \max _{y \in V} \varphi(y)$. Hence, by definition, $\pi^{*}\left(\delta_{p}\right)(\varphi) \geq \max _{y \in V} \varphi(y)$. On the other hand, for any $\epsilon>0$, choose a small neighborhood $U_{\epsilon}$ of $p$ so that

$$
\sup _{y \in \pi^{-1}\left(U_{\epsilon}\right)} \varphi(y) \leq \epsilon+\max _{y \in V} \varphi(y) .
$$

It follows that $\sup _{U_{\epsilon}} \pi_{*}(\varphi) \leq \epsilon+\max _{y \in V} \varphi(y)$. Since $\pi_{*}(\varphi)$ is continuous on $X \backslash\{p\}$, it follows by elementary set-theoretic topology that we can find a continuous function $\psi$ on $X$ so that $\psi \geq \pi_{*}(\varphi)$ and $\sup _{U_{\epsilon}} \psi \leq \epsilon+\max _{y \in V} \varphi(y)$. It follows that $\pi^{*}\left(\delta_{p}\right)(\varphi) \leq$ $\epsilon+\max _{y \in V} \varphi(y)$. Since $\epsilon$ is an arbitrary positive number, we conclude from the above discussion that $\pi^{*}\left(\delta_{p}\right)(\varphi)=\max _{y \in V} \varphi(y)$. Similarly, we can show that $\pi^{*}\left(\delta_{p}\right)(A)=$ $\delta_{p}(\pi(A \cap Y))$.

Given $f: X \rightarrow Y$ a dominant meromorphic map between compact complex varieties and $\mu$ a positive strong submeasure on $X$, we can assume without loss of generality that $X$ is smooth, by using Hironaka's resolution of singularities. By part (ii) of Theorem 1.2 and its proof, to describe $f_{*}(\mu)$ it suffices to describe $\pi^{*}(\mu)$ where $\pi: Z \rightarrow X$ is a blowup at a smooth center and $\mu$ is a measure. The following result addresses this question.

THEOREM 1.3. Let $\pi: Z \rightarrow X$ be the blowup of $X$ at an irreducible smooth subvariety $A \subset X$. Let $\varphi \in C^{0}(Z)$. Let $\mu$ be a positive measure on $X$, and decompose $\mu=\mu_{1}+\mu_{2}$ where $\mu_{1}$ has no mass on $A$ and $\mu_{2}$ has support on A. Then $\pi^{*}\left(\mu_{1}\right)$ is a positive measure 
on $Z,\left.\pi_{*}(\varphi)\right|_{A}$ is continuous, and we have

$$
\pi^{*}(\mu)(\varphi)=\pi^{*}\left(\mu_{1}\right)(\varphi)+\mu_{2}\left(\left.\pi_{*}(\varphi)\right|_{A}\right)
$$

Moreover, an explicit choice of the collection $\mathcal{G}$ in part (2) of Theorem 2.1 for $\pi^{*}(\mu)$ will be explicitly described in the proof.

The second main idea of this paper, to deal with invariant positive strong submeasures, is as follows. One can start from a positive strong submeasure $\mu_{0}$, and perform Cesaro's process on the pushforward iterates $\left(f_{*}\right)^{n}\left(\mu_{0}\right)$. The cluster points $\mu_{\infty}$ are now in general not invariant (as in the case of continuous selfmaps of compact metric spaces), the reason being that the pushforward of continuous open-dense defined selfmaps $f: X \rightarrow X$ on positive strong submeasures is not continuous, as mentioned in the previous paragraph. It turns out, however, there is a canonical way, using a type of min-max principle, to assign to any such cluster point $\mu_{\infty}$ an invariant positive strong submeasure. Here is the main result.

THEOREM 1.4. Let $f: X \rightarrow X$ be a continuous open-dense defined map on a compact metric space $X$. Let $0 \neq \mu_{0} \in S M^{+}(X)$.

(1) Let $\mu_{0}$ be a positive strong submeasure on $X$, and $\mu$ a cluster point of Cesaro's averages $(1 / n) \sum_{j=0}^{n}\left(f_{*}\right)^{j}\left(\mu_{0}\right)$. Then $f_{*}(\mu) \geq \mu$.

(2) If $f_{*}\left(\mu_{0}\right) \leq \mu_{0}$, then the set $\left\{\mu \in S M^{+}(X): \mu \leq \mu_{0}, f_{*}(\mu)=\mu\right\}$ is non-empty and has a largest element, denoted by $\operatorname{Inv}\left(\leq \mu_{0}\right)$. Moreover, $\operatorname{Inv}\left(\leq \mu_{0}\right)=$ $\lim _{n \rightarrow \infty}\left(f_{*}\right)^{n}\left(\mu_{0}\right)$.

(3) If $f_{*}\left(\mu_{0}\right) \geq \mu_{0}$, then the set $\left\{\mu \in S M^{+}(X): \mu \geq \mu_{0}, f_{*}(\mu)=\mu\right\}$ is non-empty and has a smallest element, denoted by $\operatorname{Inv}\left(\geq \mu_{0}\right)$.

Assume that $f: X \rightarrow X$ is a continuous open-dense defined selfmap. To study further dynamical properties, we need to be able to compose $f$ with itself any finite number of times, in analogy to holomorphic maps or dominant meromorphic maps. Here we follow the idea of S. Friedland. We look at the closure $\Gamma_{f, \infty}$ of the graph of $f: \operatorname{OpenDom}(f) \rightarrow X$ in the compact metric space $X^{\mathbb{N}}$.

Definition 1.5. Let $f: X \rightarrow Y$ be a continuous open-dense defined map. We say that $f$ is good with respect to iterates if the set $\Omega_{f, \infty}:=\left\{x \in \operatorname{OpenDom}(f): f^{n}(x) \notin I(f)\right.$ for all $n \in \mathbb{N}\}$ is dense in $X$ and $I_{\infty}(f)=X \backslash \Omega_{f, \infty}$ is nowhere dense.

Note that this notion applies to dominant meromorphic selfmaps of compact complex varieties, as well as to continuous maps $f: U \rightarrow U$ where $U \subset X$ is open-dense.

In this case, we can define the infinity graph $\Gamma_{f, \infty}$ as the closure in $X^{\mathbb{N}}$ (with product topology) of $\left\{\left(x, f(x), f^{2}(x), f^{3}(x), \ldots\right): x \in \Omega_{f, \infty}\right\}$. By Tikhonov's theorem, $X^{\mathbb{N}}$ is compact Hausdorff; moreover, it is a compact metric space with the metric

$$
d\left(\left(x_{1}, x_{2}, \ldots\right),\left(y_{1}, y_{2}, \ldots\right)\right):=\sum_{i=1}^{\infty} d\left(x_{i}, y_{i}\right) / 2^{i},
$$


where $d\left(x_{i}, y_{i}\right)$ is the given metric on $X$. Hence $\Gamma_{f, \infty}$ is itself a compact metric space. On $X^{\mathbb{N}}$ there is a natural shifting map $\sigma\left(x_{1}, x_{2}, x_{3}, \ldots\right)=\left(x_{2}, x_{3}, \ldots\right)$, which is continuous. It is easy to check that $\sigma\left(\Gamma_{f, \infty}\right)=\Gamma_{f, \infty}$, and we denote $\sigma_{f}:=\left.\sigma\right|_{\Gamma_{f, \infty}}: \Gamma_{f, \infty} \rightarrow \Gamma_{f, \infty}$. We can reduce several questions about $f$ to questions about $\sigma_{f}$, for example by defining $h_{\text {top }}(f):=h_{\text {top }}\left(\sigma_{f}\right)$ where the right-hand side is the usual definition of the topological entropy for a continuous map on a compact metric space. By the variational principle, the topological entropy of $\sigma_{f}$ can be determined from knowledge on invariant measures of $\sigma_{f}$. As an application of the results obtained so far, in $\S 3$ we show that there are some close relations between invariant submeasures of $\sigma_{f}$ and of $f$.

The plan of this paper is as follows. In $\S 2$ we collect some basic properties of strong submeasures. In particular, we define and prove properties of the pushforward and pullback of positive strong submeasures for open-dense defined maps. In $\S 3$ we prove the existence and some properties of invariant positive strong submeasures. In the final section we give some applications to dynamics of dominant meromorphic maps of compact Kähler surfaces.

\section{Strong submeasures}

In this section we collect some basic properties of strong submeasures on a compact metric space $X$ which are needed to establish basic ergodic properties, and their pushforward by continuous maps $f: U \rightarrow Y$, where $U \subset X$ is a dense open subset and $Y$ is another compact metric space. If $f$ is a proper covering map of finite degree to its image $f(U)$, we can also define a pullback operator on positive strong submeasures.

2.1. Strong submeasures. Let $X$ be a compact metric space. We recall the notation from the introduction: $M(X)$ the set of signed measures on $X, M^{+}(X)$ the set of positive measures on $X, S M(X)$ the set of strong submeasures on $X$ and $S M^{+}(X)$ the set of positive strong submeasures on $X$.

By a simple application of the Hahn-Banach extension theorem (see [17]) and Riesz representation theorem (see [18]), we have the following characterization, whose proof is omitted, of strong submeasures and positive strong submeasures.

THEOREM 2.1. Let $X$ be a compact metric space, and $\mu: C^{0}(X) \rightarrow \mathbb{R}$ an operator.

(1) $\mu$ is a strong submeasure if and only if there is a non-empty collection $\mathcal{G}$ of signed measures $\chi=\chi^{+}-\chi^{-}$where $\chi^{ \pm}$are measures on $X$ so that $\sup _{\chi=\chi^{+}-\chi^{-} \in \mathcal{G}} \chi^{ \pm}(1)<\infty$, and

$$
\mu(\varphi)=\sup _{\chi \in \mathcal{G}} \chi(\varphi)
$$

for all continuous functions $\varphi$.

(2) $\quad \mu$ is a positive strong submeasure if and only if there is a non-empty collection $\mathcal{G}$ of (positive) measures on $X$ so that $\sup _{\chi \in \mathcal{G}} \chi(1)<\infty$, and

$$
\mu(\varphi)=\sup _{\chi \in \mathcal{G}} \chi(\varphi)
$$

for all continuous functions $\varphi$.

The next paragraphs discuss the natural topology on the space of strong submeasures. 
Definition 2.2. We say that a sequence $\mu_{1}, \mu_{2}, \ldots \in S M(X)$ weakly converges to $\mu \in S M(X)$ if $\sup _{n}\left\|\mu_{n}\right\|<\infty$ and

$$
\lim _{n \rightarrow \infty} \mu_{n}(\varphi)=\mu(\varphi)
$$

for all $\varphi \in C^{0}(X)$. We use the notation $\mu_{n} \rightarrow \mu$ to denote that $\mu_{n}$ weakly converges to $\mu$.

If $\mu_{1}, \mu_{2}: C^{0}(X) \rightarrow \mathbb{R}$, we define $\max \left\{\mu_{1}, \mu_{2}\right\}: C^{0}(X) \rightarrow \mathbb{R}$ by the formula $\max \left\{\mu_{1}, \mu_{2}\right\}(\varphi)=\max \left\{\mu_{1}(\varphi), \mu_{2}(\varphi)\right\}$. Theorem 2.3 shows that submeasures also have properties similar to measures, such as weak compactness.

For later use, we recall that for a compact subset $A \subset X$, we have [12]

$$
\mu(A)=\inf _{\phi \in C^{0}\left(X, \geq 1_{A}\right)} \mu(\phi) .
$$

Here $1_{A}: X \rightarrow\{0,1\}$ is the characteristic function of $A$, that is, $1_{A}(x)$ equals 1 if $x \in A$ and equals 0 otherwise, $C^{0}(X)$ is the space of continuous functions from $X$ into $\mathbb{R}$, and for any bounded function $g: X \rightarrow \mathbb{R}$ we use the notation

$$
C^{0}(X, \geq g)=\left\{\phi \in C^{0}(X): \phi \geq g\right\} .
$$

Moreover, for any open set $B \subset X$ we have [12]

$$
\mu(B)=\sup _{A \operatorname{compact} \subset B} \mu(A) .
$$

Like measures, positive strong submeasures give rise naturally to set functions. On a compact metric space $X$, recall that a function $g: X \rightarrow \mathbb{R}$ is upper-semicontinuous if there is a sequence of continuous functions $g_{n}$ on $X$ decreasing to $g$; see [2]. Hence, if $\mu$ is a measure, we have by Lebesgue and Levi's monotone convergence theorem in integration theory that

$$
\mu(g)=\lim _{n \rightarrow \infty} \mu\left(g_{n}\right)=\inf _{\varphi \in C^{0}(X, \geq g)} \mu(\varphi) .
$$

Inspired by this and (2.4), if $\mu$ is an arbitrary strong submeasure, we define for any upper-semicontinuous function $g$ on $X$ the value

$$
E(\mu)(g):=\inf _{\varphi \in C^{0}(X, \geq g)} \mu(\varphi) \in[-\infty, \infty) .
$$

Then for a closed set $A \subset X$, we define $\mu(A):=E(\mu)\left(1_{A}\right)$ where $1_{A}$ is the characteristic function of $A$. If $\mu$ is positive, we always have $\mu(A) \geq 0$. Then, for an open subset $B \subset X$, following (2.6) we define $\mu(B):=\sup \{\mu(A): A$ compact $\subset B\}$. Theorem 2.4 proves some basic properties of this operator, similar to those of submeasures.

If we have a positive strong submeasure $\mu$, and define for any Borel set $A \subset X$ the number $\tilde{\mu}(A)=\inf \{\mu(B): B$ open, $A \subset B\}$, then we see easily from part (4) of Theorem 2.4 that: (i) $\tilde{\mu}(\emptyset)=0$, (ii) $\tilde{\mu}\left(A_{1}\right) \leq \tilde{\mu}\left(A_{2}\right)$ for all Borel sets $A_{1} \subset A_{2}$, and (iii) $\tilde{\mu}\left(A_{1} \cup A_{2}\right) \leq \tilde{\mu}\left(A_{1}\right)+\tilde{\mu}\left(A_{2}\right)$. Such $\tilde{\mu}$ are known in the literature as submeasures (see, for example, [19]), and hence it is justified to call our objects $\mu$ positive strong submeasures. 
We have the following basic properties of strong submeasures, whose proof is easy and hence omitted.

THEOREM 2.3. Let $X$ be a compact metric space.

(1) Weak-compactness. Let $\mu_{1}, \mu_{2}, \ldots$ be a sequence in $S M(X)$ such that $\sup _{n}\left\|\mu_{n}\right\|<$ $\infty$. Then there is a subsequence $\left\{\mu_{n(k)}\right\}_{k=1,2, \ldots}$ which weakly converges to some $\mu \in$ $S M(X)$. If, moreover, $\mu_{n} \in S M^{+}(X)$, then so is $\mu$.

(2) If $\mu \in S M^{+}(X)$, then $\|\mu\|=\max \{|\mu(1)|,|\mu(-1)|\}$.

(3) If $\mu_{1}, \mu_{2} \in S M(X)$ then $\max \left\{\mu_{1}, \mu_{2}\right\}$ and $\mu_{1}+\mu_{2}$ are also in $S M(X)$. If $\mu_{1}, \mu_{2} \in$ $S M^{+}(X)$ then $\max \left\{\mu_{1}, \mu_{2}\right\}$ and $\mu_{1}+\mu_{2}$ are also in $S M^{+}(X)$.

The next result is the main technical result to deal with the pushforward of measures by a map defined only on a dense open set. Denote by $B U S(X)$ the set of all bounded upper-semicontinuous functions on $X$.

THEOREM 2.4. Let $X$ be a compact metric space and $\mu \in S M(X)$. Let $E(\mu): B U S(X) \rightarrow$ $[-\infty, \infty)$ be defined as in (2.7). Assume that $E(\mu)(0)$ is finite.

(1) For all $\varphi \in B U S(X)$, the value $E(\mu)(\varphi)$ is finite. Moreover, $E(\mu)(0)=0$ and $E(\mu)(-1) \geq-\mu(1)$.

(2) Extension. If $\mu$ is positive, then for all $\varphi \in C^{0}(X)$ we have $E(\mu)(\varphi)=\mu(\varphi)$.

(3) Moreover, $E(\mu)$ satisfies the following properties.

(i) Sub-linearity. $E(\mu)\left(\varphi_{1}+\varphi_{2}\right) \leq E(\mu)\left(\varphi_{1}\right)+E(\mu)\left(\varphi_{2}\right)$ and $E(\mu)(\lambda \varphi)=$ $\lambda E(\mu)(\varphi)$ for $\varphi_{1}, \varphi_{2}, \varphi \in B U S(X)$ and a non-negative constant $\lambda$.

(ii) Positivity. $E(\mu)\left(\varphi_{1}\right) \geq E(\mu)\left(\varphi_{2}\right)$ for all $\varphi_{1}, \varphi_{2} \in B U S(X)$ satisfying $\varphi_{1} \geq \varphi_{2}$.

(iii) Boundedness. There is a constant $C>0$ so that for all $\varphi \in B U S(X)$ we have $|E(\mu)(\varphi)| \leq C\|\varphi\|_{L^{\infty}}$. The least such constant $C$ is in fact $\|\mu\|$.

(4) If $A_{1}, A_{2}$ are closed subsets of $X$ then $\mu\left(A_{1} \cup A_{2}\right) \leq \mu\left(A_{1}\right)+\mu\left(A_{2}\right)$. Likewise, if $B_{1}, B_{2}$ are open subsets of $X$ then $\mu\left(B_{1} \cup B_{2}\right) \leq \mu\left(B_{1}\right)+\mu\left(B_{2}\right)$.

Proof of Theorem 2.4. (1) We first observe that, for all $\varphi \in C^{0}(X, \geq 0)$, we have $\mu(\varphi) \geq 0$. (Note that, as observed in $\S 1$, this fact alone does not imply that $\mu$ is a positive strong submeasure.) In fact, otherwise, there would be $\varphi_{0} \in C^{0}(X, \geq 0)$ so that $\mu\left(\varphi_{0}\right)<0$. Then by the definition of $E(\mu)$ and sub-linearity of $\mu$ we have

$$
E(\mu)(0) \leq \inf _{n \in \mathbb{N}} \mu\left(n \varphi_{0}\right)=\inf _{n \in \mathbb{N}} n \mu\left(\varphi_{0}\right)=-\infty,
$$

which contradicts the assumption that $E(\mu)(0)$ is finite.

Therefore, if $\varphi \in C^{0}(X, \geq 0)$, we obtain

$$
0 \leq \inf _{\psi \in C^{0}(X, \geq \varphi)} \mu(\psi) \leq \mu(\varphi) .
$$

Therefore, for these functions $\varphi$ we have that $E(\mu)(\varphi)$ is a finite number. In particular, $0 \leq E(\mu)(1) \leq \mu(1)$.

Next, we observe that if $\varphi_{1}, \varphi_{2} \in B U S(X)$ such that either $E(\mu)\left(\varphi_{1}\right)$ or $E(\mu)\left(\varphi_{2}\right)$ is finite, then the proof of (3)(i) is still valid and gives $E(\mu)\left(\varphi_{1}+\varphi_{2}\right) \leq E(\mu)\left(\varphi_{1}\right)+$ $E(\mu)\left(\varphi_{2}\right)$. Applying this sub-linearity to $\varphi_{1}=\varphi_{2}=0$, we obtain $E(\mu)(0)=E(\mu)(0+$ 
$0) \leq 2 E(\mu)(0)$, which implies $E(\mu)(0) \geq 0$. On the other hand, $E(\mu)(0) \leq \mu(0)=0$. Therefore, $E(\mu)(0)=0$.

Since $E(\mu)(1)$ is finite, applying the above sub-linearity for $\varphi_{1}=1$ and $\varphi_{2}=-1$, we obtain $0=E(0)=E(\mu)(1+(-1)) \leq E(\mu)(1)+E(\mu)(-1)$. Therefore, $E(\mu)(-1) \geq$ $-E(\mu)(1) \geq-\mu(1)$.

Finally, applying the proof of part (3)(iii), we deduce that for all $\varphi \in B U S(X)$, the number $E(\mu)(\varphi)$ is finite.

(2) Let $\varphi \in C^{0}(X)$, and choose any $\psi \in C^{0}(X, \geq \varphi)$. Since $\mu$ is positive, we have by definition that $\mu(\psi) \geq \mu(\varphi)$. Since $\varphi$ is itself contained in $C^{0}(X, \geq \varphi)$, it follows that

$$
E(\mu)(\varphi)=\inf _{\psi \in C^{0}(X, \geq \varphi)} \mu(\psi)=\mu(\varphi) .
$$

(3) Let $\varphi, \varphi_{1}, \varphi_{2} \in B U S(X)$.

(i) If $\psi_{1} \in C^{0}\left(X, \geq \varphi_{1}\right)$ and $\psi_{2} \in C^{0}\left(X, \geq \varphi_{2}\right)$ then $\psi_{1}+\psi_{2} \in C^{0}(X, \geq$ $\left.\varphi_{1}+\varphi_{2}\right)$. Therefore, by sub-linearity of $\mu$,

$$
E(\mu)\left(\varphi_{1}+\varphi_{2}\right)=\inf _{\psi \in C^{0}\left(X, \geq \varphi_{1}+\varphi_{2}\right)} \mu(\psi) \leq \mu\left(\psi_{1}+\psi_{2}\right) \leq \mu\left(\psi_{1}\right)+\mu\left(\psi_{2}\right) .
$$

We can choose $\psi_{1}$ and $\psi_{2}$ so that $\mu\left(\psi_{1}\right)$ is arbitrarily close to $E(\mu)\left(\varphi_{1}\right)$ and $\mu\left(\psi_{2}\right)$ is arbitrarily close to $E(\mu)\left(\varphi_{2}\right)$, and from that obtain the desired conclusion $E(\mu)\left(\varphi_{1}+\varphi_{2}\right) \leq E(\mu)\left(\varphi_{1}\right)+E(\mu)\left(\varphi_{2}\right)$. The other part of (i) is easy to check.

(ii) If $\varphi_{1} \geq \varphi_{2}$ then $C^{0}\left(X, \geq \varphi_{1}\right) \subset C^{0}\left(X\right.$, $\left.\geq \varphi_{2}\right)$. From this the conclusion follows.

(iii) We observe that we can find $\psi \in C^{0}(X, \geq \varphi)$ so that $\|\psi\|_{L^{\infty}}=\|\varphi\|_{L^{\infty}}$, simply by defining $\psi=\max \left\{\min \left\{\psi_{0},\|\varphi\|_{L^{\infty}}\right\},-\|\varphi\|_{L^{\infty}}\right\}$ for any $\psi_{0} \in$ $C^{0}(X, \geq \varphi)$. Then

$$
E(\mu)(\varphi) \leq \mu(\psi) \leq\|\mu\| \times\|\psi\|_{L^{\infty}}=\|\mu\| \times\|\varphi\|_{L^{\infty}} .
$$

By the positivity of $E(\mu)$ in (ii), we have

$$
E(\mu)(\varphi) \geq\|\varphi\|_{L^{\infty}} E(\mu)(-1),
$$

and hence $|E(\mu)(\varphi)| \leq \max \{|E(\mu)(-1)|,\|\mu\|\}=\|\mu\|$. In the last equality we used that (1) and positivity imply $-\mu(1) \leq E(\mu)(-1) \leq E(\mu)(0)=0$.

(4) By definition, we have for closed subsets $A_{1}, A_{2} \subset X$,

$$
\mu(A)=E(\mu)\left(1_{A_{1} \cup A_{2}}\right) \leq E(\mu)\left(1_{A_{1}}+1_{A_{2}}\right) \leq E(\mu)\left(1_{A_{1}}\right)+E(\mu)\left(1_{A_{2}}\right)=\mu\left(A_{1}\right)+\mu\left(A_{2}\right) .
$$

In the first inequality we used $1_{A_{1} \cup A_{2}} \leq 1_{A_{1}}+1_{A_{2}}$ and the positivity of $E(\mu)$. In the second inequality we used the sub-linearity of $E(\mu)$.

If $B_{1}, B_{2}$ are open subsets of $X$ and $A \subset B_{1} \cup B_{2}$ is closed in $X$, then since $X$ is compact metric we can find closed subsets $A_{1}, A_{2}$ of $X$ so that $A_{1} \subset B_{1}, A_{2} \subset B_{2}$ and $A_{1} \cup A_{2}=A$. To this end, for each $x \in A$, we choose an open ball $B\left(x, r_{x}\right)$ (in the given metric on $X$ ) where $r_{x}>0$ is chosen as follows: if $x \in B_{1}$ then the closed ball $\overline{B\left(x, r_{x}\right)}$ belongs to $B_{1}$, if $x \in B_{2}$ then the closed ball $\overline{B\left(x, r_{x}\right)}$ belongs to $B_{2}$, and if $x \in B_{1} \cap B_{2}$ then the closed ball $\overline{B\left(x, r_{x}\right)}$ belongs to $B_{1} \cap B_{2}$. Since $A$ is compact, there 
are a finite number of such balls covering $A: A \subset \bigcup_{i=1}^{m} B\left(x_{i}, r_{i}\right)$. Then the choice of $A_{1}=A \cap\left(\bigcup_{x_{i} \in B_{1}} \overline{B\left(x_{i}, r_{i}\right)}\right)$ and $A_{2}=A \cap\left(\bigcup_{x_{i} \in B_{2}} \overline{B\left(x_{i}, r_{i}\right)}\right)$ satisfies the requirement. Then from the above sub-linearity of $\mu$ for compact sets and the definition, we have also sub-linearity for open sets $\mu\left(B_{1} \cup B_{2}\right) \leq \mu\left(B_{1}\right)+\mu\left(B_{2}\right)$.

2.2. Pushforward of positive strong submeasures. Throughout this subsection we let $f: X \rightarrow Y$ be a continuous open-dense defined map, where $X$ and $Y$ are compact metric spaces. In this subsection we discuss several results concerning the pushforward of positive strong submeasures on $X$ by $f$. The key to this is the next result, whose proof is simple and hence is left to the reader.

Proposition 2.5. Let $X$ be a compact metric space, $U \subset X$ an open-dense set, and $g: U \rightarrow \mathbb{R}$ a bounded upper semicontinuous function. Define $E(g): X \rightarrow \mathbb{R}$ as follows. If $x \in U$ then $E(g)(x):=g(x)$, and if $x \in X \backslash U$ then

$$
E(g)(x):=\limsup _{y \in U, y \rightarrow x} g(y) .
$$

Then:

(1) $E(g)$ is a bounded upper-semicontinuous function, and $\left.E(g)\right|_{U}=g$. In other words, $E(g)$ is a bounded upper-semicontinuous extension of $g$.

(2) If $g$ is continuous on $U, U_{1} \subset U$ is another open-dense set of $X$ and $g_{1}=\left.g\right|_{U_{1}}$, then $E\left(g_{1}\right)=E(g)$.

(3) Moreover, $E\left(g_{1}+g_{2}\right) \leq E\left(g_{1}\right)+E\left(g_{2}\right)$ for any $g_{1}, g_{2}: U \rightarrow \mathbb{R}$ bounded uppersemicontinuous functions.

Remark. On the other hand, if $g$ is not continuous on $U$ then it is easy to construct examples for which the conclusion of part (2) in the proposition does not hold. Let $\Gamma_{f} \subset X \times Y$ be the closure of the graph $\{(x, f(x)): x \in \operatorname{OpenDom}(f)\}$. Then, with the induced topology from $X \times Y, \Gamma_{f}$ is a compact metric space itself. We have two canonical projections $\pi_{X}, \pi_{Y}: X \times Y \rightarrow X, Y$, whose restrictions to $\Gamma_{f}$ are denoted $\pi_{X, f}, \pi_{Y, f}$. If we let $U \subset$ OpenDom $(f)$ be any open-dense subset, and since $V=\pi_{X, f}^{-1}(U)$, then $V$ is an open-dense subset of $\Gamma_{f}$ and we see that $\pi_{V, f}:=\left.\pi_{X, f}\right|_{V}: V \rightarrow U$ is a homeomorphism.

Definition 2.6. Using Proposition 2.5, we define $\left(\pi_{X, f}\right)_{*}(\phi)$, where $\phi \in C^{0}\left(\Gamma_{f}\right)$, to be the upper-semicontinuous function $E\left(\left(\pi_{V, f}\right)_{*}(\phi)\right)$ on $X$. We emphasize that it is globally defined on the whole of $X$, and is not changed if we replace $V$ (or $U$ ) by one open-dense subset of it.

Finally, we have a canonical definition of the pullback of continuous functions by $f$ : $X \rightarrow Y$.

Definition 2.7. Let $\varphi \in C^{0}(Y)$. We denote by $f^{*}(\varphi)$ the above upper-semicontinuous function $\left(\pi_{X, f}\right)_{*}\left(\pi_{Y, f}^{*}(\phi)\right)$.

Note that when $X, Y$ are smooth complex varieties, $f: X \rightarrow Y$ a meromorphic map, and $\varphi$ is a continuous quasi-plurisubharmonic function on $Y$ (for example, if $\varphi$ is a 
$C^{2}$ function), then our definition of the pullback $f^{*}(\varphi)$ above is the standard one in [16]. In this case, any continuous function $\varphi$ is a uniform limit of a sequence of $C^{2}$ functions. This observation and the following proposition, whose simple proof is omitted, justify our Definition 2.7.

Proposition 2.8. Let $f: X \rightarrow Y$ be a continuous open-dense defined map as at the beginning of this section. Assume that $\varphi_{n}$ is a sequence of continuous functions on $Y$ uniformly converging to a continuous function $\varphi$. Then $\left\{f^{*}\left(\varphi_{n}\right)\right\}$ converges uniformly to $f^{*}(\varphi)$.

Using Proposition 2.5 and the above upper-semicontinuous pushforward of functions, we can finally define, following Theorem 2.4, the following pullback operator $\pi_{X, f}^{*}$ : $S M^{+}(X) \rightarrow S M^{+}\left(\Gamma_{f}\right):$

$$
\pi_{X, f}^{*}(\mu)(\varphi):=\inf _{\psi \in C^{0}\left(X, \geq\left(\pi_{X, f}\right)_{*}(\varphi)\right)} \mu(\psi)
$$

Then, as in the case of continuous maps $g: X \rightarrow Y$ between compact metric spaces, we define $f_{*}(\mu)$, for a positive strong submeasure $\mu$, by the formula $f_{*}(\mu)=$ $\left(\pi_{Y, f}\right)_{*} \pi_{X, f}^{*}(\mu)$.

Definition 2.9. For convenience, we write here the final formula for pushing forward a strong submeasure by our maps $f: X \rightarrow Y$ :

$$
f_{*}(\mu)(\varphi):=\inf _{\psi \in C^{0}\left(X, \geq\left(\pi_{X, f}\right)_{*}\left(\pi_{Y, f}^{*}(\varphi)\right)\right)} \mu(\psi)
$$

Here is the main result of this subsection. In its proof we use the pullback of positive strong submeasures by the projection $\pi$, via pushforward by its inverse $\pi^{-1}$. For more general details about the pullback of positive strong submeasures, see the next subsection.

THEOREM 2.10. Let $X, Y$ be compact metric spaces, and $f: X \rightarrow Y$ a continuous open-dense defined map.

(1) We have $f_{*}\left(S M^{+}(X)\right) \subset S M^{+}(Y)$. Moreover, if $\mu \in S M^{+}(X)$, then $f_{*}(\mu)( \pm 1)=$ $\mu( \pm 1)$. In particular, $\left\|f_{*}(\mu)\right\|=\|\mu\|$.

(2) Assume that $g: Y \rightarrow Z$ is another continuous open-dense defined map, and there $i$ an open-dense set $U \subset \operatorname{OpenDom}(f)$ such that $f(U) \subset \operatorname{OpenDom}(g)$. For all $\mu \in S M^{+}(X)$, we have $g_{*} f_{*}(\mu) \geq(g \circ f)_{*}(\mu)$. If $f$ and $g$ are continuous on the whole of $X$ and $Y$, then equality happens.

(3) If $\mu_{n} \in S M^{+}(X)$ weakly converges to $\mu$, and $v$ is a cluster point of $f_{*}\left(\mu_{n}\right)$, then $v \leq f_{*}(\mu)$. If $f$ is continuous on the whole of $X$, then $\lim _{n \rightarrow \infty} f_{*}\left(\mu_{n}\right)=f_{*}(\mu)$.

(4) If $\mu$ is a positive measure without mass on $I(f)$, then $f_{*}(\mu)$ is the same as the usual definition.

(5) For any positive strong submeasure $\mu$, we have $f_{*}(\mu)=\sup _{\chi \in \mathcal{G}(\mu)} f_{*}(\chi)$, where $\mathcal{G}(\mu)=\{\chi: \chi$ is a measure and $\chi \leq \mu\}$.

(6) For every positive strong submeasure $\mu_{1}, \mu_{2}$, we have $f_{*}\left(\mu_{1}+\mu_{2}\right) \geq f_{*}\left(\mu_{1}\right)+$ $f_{*}\left(\mu_{2}\right)$. 
Proof of Theorem 2.10. (1) Let $\mu \in S M^{+}(X)$. We show that $f_{*}(\mu) \in S M^{+}(Y)$. Let $\varphi, \varphi_{1}, \varphi_{2} \in C^{0}(Y)$ and $0 \leq \lambda \in \mathbb{R}$.

First, we show that $f_{*}(\mu)( \pm 1)=\mu( \pm 1)$. In fact, it follows from the definition that $\left(\pi_{X, f}\right)_{*} \pi_{Y, f}^{*}( \pm 1)= \pm 1$, and hence

$$
f_{*}(\mu)( \pm 1)=\inf _{\psi \in C^{0}(X, \geq \pm 1)} \mu(\psi)=\mu( \pm 1) .
$$

Second, we show the positivity of $f_{*}(\mu)$. If $\varphi_{1} \geq \varphi_{2}$, then it can be seen from the definition that $\left(\pi_{X, f}\right)_{*} \pi_{Y, f}^{*}\left(\varphi_{1}\right) \geq\left(\pi_{X, f}\right)_{*} \pi_{Y, f}^{*}\left(\varphi_{2}\right)$. Therefore $C^{0}(X, \geq$ $\left.\left(\pi_{X, f}\right)_{*} \pi_{Y, f}^{*}\left(\varphi_{1}\right)\right) \subset C^{0}\left(X, \geq\left(\pi_{X, f}\right)_{*} \pi_{Y, f}^{*}\left(\varphi_{2}\right)\right)$, and hence it follows by definition that $f^{*}(\mu)\left(\varphi_{1}\right) \geq f^{*}(\mu)\left(\varphi_{2}\right)$.

Next, we show that $f_{*}(\mu)$ is bounded and, moreover, that $\left\|f_{*}(\nu)\right\|=\operatorname{deg}(f)\|\mu\|$. By positivity of $f_{*}(\mu)$, we have $f_{*}(\mu)\left(-\|\varphi\|_{L^{\infty}}\right) \leq f_{*}(\mu) \leq f_{*}(\mu)\left(\|\varphi\|_{L^{\infty}}\right)$. Hence $f_{*}(\nu)$ is bounded, and we conclude by Theorem 2.3.

Finally, we show the sub-linearity. The equality $f_{*}(\mu)(\lambda \varphi)=\lambda f_{*}(\mu)(\varphi)$, for $\lambda \geq 0$, follows from the fact that $\left(\pi_{X, f}\right)_{*} \pi_{Y, f}^{*}(\lambda \varphi)=\lambda\left(\pi_{X, f}\right)_{*} \pi_{Y, f}^{*}(\varphi)$ and properties of the infimum. We now prove that $f_{*}(\mu)\left(\varphi_{1}+\varphi_{2}\right) \leq f^{*}(\mu)\left(\varphi_{1}\right)+f^{*}(\mu)\left(\varphi_{2}\right)$. In fact, from Proposition 2.5 we have

$$
\left(\pi_{X, f}\right)_{*} \pi_{Y, f}^{*}\left(\varphi_{1}+\varphi_{2}\right) \leq\left(\pi_{X, f}\right)_{*} \pi_{Y, f}^{*}\left(\varphi_{1}\right)+\left(\pi_{X, f}\right)_{*} \pi_{Y, f}^{*}\left(\varphi_{2}\right),
$$

and hence if $\psi_{1} \in C^{0}\left(X, \geq\left(\pi_{X, f}\right)_{*} \pi_{Y, f}^{*}\left(\varphi_{1}\right)\right)$ and $\psi_{2} \in C^{0}\left(X, \geq\left(\pi_{X, f}\right)_{*} \pi_{Y, f}^{*}\left(\varphi_{2}\right)\right)$ then $\psi_{1}+\psi_{2} \in C^{0}\left(X, \geq\left(\pi_{X, f}\right)_{*} \pi_{Y, f}^{*}\left(\varphi_{1}+\varphi_{2}\right)\right)$. Hence, by definition,

$$
f_{*}(\mu)\left(\varphi_{1}+\varphi_{2}\right) \leq \mu\left(\psi_{1}+\psi_{2}\right) \leq \mu\left(\psi_{1}\right)+\mu\left(\psi_{2}\right) .
$$

In the second inequality we used the sub-linearity of $\mu$. If we choose $\psi_{1}$ and $\psi_{2}$ so that $\mu\left(\psi_{1}\right)$ is close to $f_{*}(\mu)\left(\varphi_{1}\right)$ and $\mu\left(\psi_{2}\right)$ is close to $f_{*}(\mu)\left(\varphi_{2}\right)$, then we see that $f_{*}(\mu)\left(\varphi_{1}+\right.$ $\left.\varphi_{2}\right) \leq f_{*}(\mu)\left(\varphi_{1}\right)+f_{*}(\mu)\left(\varphi_{2}\right)$ as desired.

(2) By definition, we have

$$
(g \circ f)_{*}(\mu)(\varphi)=\inf _{\psi \in C^{0}\left(X, \geq(g \circ f)^{*}(\varphi)\right)} \mu(\psi) .
$$

Here we recall that $(g \circ f)^{*}(\varphi)$ is the upper-semicontinuous pullback of $\varphi$ by $g \circ f$.

On the other hand,

$$
g_{*} f_{*}(\mu)(\varphi)=\inf _{\psi_{1} \in C^{0}\left(Y, \geq g^{*}(\varphi)\right)} f_{*}(\mu)\left(\psi_{1}\right)=\inf _{\psi_{1} \in C^{0}\left(Y, \geq g^{*}(\varphi)\right)} \inf _{\psi_{2} \in C^{0}\left(X, \geq f^{*}\left(\psi_{1}\right)\right)} \mu\left(\psi_{2}\right) .
$$

Then it follows by the proof of part (2) of Proposition 2.5 that whenever $\psi_{1} \in C^{0}(Y, \geq$ $\left.g^{*}(\varphi)\right)$ and $\psi_{2} \in C^{0}\left(Y, \geq f^{*}\left(\psi_{1}\right)\right)$, we have $\psi_{2} \in C^{0}\left(X, \geq(g \circ f)^{*}(\varphi)\right)$. From this, we get $g_{*} f_{*}(\varphi)(\nu) \geq(g \circ f)_{*}(\mu)(\varphi)$.

When $f$ and $g$ are continuous on the whole of $X$ and $Y$ and $\varphi$ is continuous, we have that $g^{*}(\varphi), f^{*}\left(g^{*}(\varphi)\right)$ and $(g \circ f)^{*}(\varphi)$ are all continuous functions. Then, using the positivity of $\mu$, we can easily see that

$$
(g \circ f)_{*}(\mu)(\varphi)=\mu\left(f^{*} g^{*}(\varphi)\right)=f_{*}(\mu)\left(g^{*}(\varphi)\right)=g_{*} f_{*}(\mu)(\varphi) .
$$


(3) It is enough to show that, for all $\varphi \in C^{0}(Y)$,

$$
\limsup _{n \rightarrow \infty} \inf _{\psi \in C^{0}\left(X, \geq f^{*}(\varphi)\right)} \mu_{n}(\psi) \leq \inf _{\psi \in C^{0}\left(X, \geq f^{*}(\varphi)\right)} \mu(\psi) .
$$

If we choose $\psi_{0} \in C^{0}\left(X, \geq f^{*}(\varphi)\right)$ so that $\mu\left(\psi_{0}\right)$ is close to $f_{*}(\mu)(\varphi)$, then from $\mu_{n}\left(\psi_{0}\right) \rightarrow \mu\left(\psi_{0}\right)$ we obtain the conclusion.

If $f$ is continuous on the whole of $X$, then $f^{*}(\varphi)$ is itself a continuous function. Then it is easy to see that $\lim _{n \rightarrow \infty} f_{*}\left(\mu_{n}\right)(\varphi)=f_{*}(\mu)(\varphi)$.

(4) The upper-semicontinuous pullback $f^{*}(\varphi)$ of a function $\varphi \in C^{0}(Y)$ is continuous on the open set $U=X \backslash I(f)$. Therefore, by choosing a small open neighborhood $U_{1}$ of $I(f)$ and a partition of unity subordinate to $U$ and $U_{1}$, it is easy to find for any $U_{2} \subset \subset U$ a $\psi \in C^{0}\left(X, \geq f^{*}(\varphi)\right)$ so that $\left.\psi\right|_{U_{2}}=\left.f^{*}(\varphi)\right|_{U_{2}}$. From this and the assumption that $\mu$ has no mass on $I(f)$, the conclusion follows.

(5) As mentioned before the statement of the theorem, since $\pi_{X, f}: \Gamma_{f} \backslash \pi_{X, f}^{-1}(I(f)) \rightarrow$ $X \backslash I(f)$ is a homeomorphism, we can define the pullback of a positive strong submeasure on $X$ (as a positive strong submeasure on $\Gamma_{f}$ ) as the pushforward of the continuous open-dense defined map $\pi_{X, f}^{-1}: X \rightarrow \Gamma_{f}$. We have for any positive strong submeasure $\mu$ that $f_{*}(\mu)=\left(\pi_{Y, f}\right)_{*} \pi_{X, f}^{*}(\mu)$. It is easy to check that the conclusion holds for $\pi_{Y, f}$, and hence to prove the result it suffices to prove that $\pi_{X, f}^{*}(\mu)=\sup _{\chi \in \mathcal{G}(\mu)} \pi^{*}(\chi)$.

Since $\pi_{X, f}^{*}(\mu) \geq \pi_{X, f}^{*}(\chi)$ for all $\chi \in \mathcal{G}(\mu)$, it follows that $\pi_{X, f}^{*}(\mu) \geq \sup _{\chi \in \mathcal{G}(\mu)} \pi_{X, f}^{*}(\chi)$. Now we will prove the reverse inequality. To this end, it suffices to show that, for any measure $\chi^{\prime} \leq \pi^{*}(\mu)$, there is a measure $\chi \leq \mu$ so that $\chi^{\prime} \leq \pi^{*}(\chi)$.

We first show that $\left(\pi_{X, f}\right)_{*} \pi_{X, f}^{*}(\mu)=\mu$. In fact, if $\varphi \in C^{0}(X)$ then $\pi_{X, f}^{*}(\varphi) \in C^{0}(Z)$ and $\varphi=\left(\pi_{X, f}\right)_{*} \pi_{X, f}^{*}(\varphi)$. Hence, by definition,

$$
\left(\pi_{X, f}\right)_{*} \pi_{X, f}^{*}(\mu)(\varphi)=\pi_{X, f}^{*}(\mu)\left(\pi_{X, f}^{*}(\varphi)\right)=\mu\left(\left(\pi_{X, f}\right) * \pi_{X, f}^{*}(\varphi)\right)=\mu(\varphi) .
$$

Hence $\left(\pi_{X, f}\right)_{*} \pi_{X, f}^{*}(\mu)=\mu$ as desired.

Now if $\chi^{\prime}$ is any measure on $Z$, then $\chi=\left(\pi_{X, f}\right)_{*}\left(\chi^{\prime}\right)$ is a measure on $X$. If, moreover, $\chi^{\prime} \leq \pi_{X, f}^{*}(\mu)$, then

$$
\chi=\left(\pi_{X, f}\right)_{*}\left(\chi^{\prime}\right) \leq\left(\pi_{X, f}\right)_{*} \pi_{X, f}^{*}(\mu)=\mu .
$$

To conclude the proof, we will show that $\pi_{X, f}^{*}(\chi) \geq \chi^{\prime}$. To this end, let $\varphi \in C^{0}(Z)$. We will show that $\pi_{X, f}^{*}(\chi)(\varphi) \geq \chi^{\prime}(\varphi)$. By definition, the value of the positive strong submeasure $\pi_{X, f}^{*}(\chi)$ at $\varphi$ is defined as $\pi_{X, f}^{*}(\chi)(\varphi)=\inf _{\psi \in C^{0}\left(X, \geq\left(\pi_{X, f}\right)_{*}(\varphi)\right)} \chi(\psi)$, and since $\chi=$ $\left(\pi_{X, f}\right)_{*}\left(\chi^{\prime}\right)$ the right-hand side is equal to $\inf _{\psi \in C^{0}\left(X, \geq\left(\pi_{X, f}\right)_{*}(\varphi)\right)} \chi^{\prime}\left(\pi_{X, f}^{*}(\psi)\right) \geq \chi^{\prime}(\varphi)$. The latter follows from the fact that $\chi^{\prime}$ is a positive strong submeasure and that for all $\psi \in C^{0}\left(X, \geq\left(\pi_{X, f}\right)_{*}(\varphi)\right)$ we have $\pi_{X, f}^{*}(\psi) \geq \varphi$.

(6) Let $\varphi$ be a continuous function on $X$. We then have by part (5), using $\mathcal{G}\left(\mu_{1}\right)+$ $\mathcal{G}\left(\mu_{2}\right) \subset \mathcal{G}\left(\mu_{1}+\mu_{2}\right)$, that

$$
\begin{aligned}
f_{*}\left(\mu_{1}+\mu_{2}\right)(\varphi) & =\sup _{\nu \in \mathcal{G}\left(\mu_{1}+\mu_{2}\right)} \inf _{\psi \in C^{0}\left(\geq f^{*}(\varphi)\right)} v(\psi) \\
& \geq \sup _{\nu_{1} \in \mathcal{G}\left(\mu_{1}\right), \nu_{2} \in \mathcal{G}\left(\mu_{2}\right)} \inf _{\psi \in C^{0}\left(\geq f^{*}(\varphi)\right)}\left(v_{1}+v_{2}\right)(\psi)
\end{aligned}
$$




$$
\begin{aligned}
& \geq \sup _{v_{1} \in \mathcal{G}\left(\mu_{1}\right), \nu_{2} \in \mathcal{G}\left(\mu_{2}\right)}\left[\inf _{\psi \in C^{0}\left(\geq f^{*}(\varphi)\right)} v_{1}(\psi)+\inf _{\psi \in C^{0}\left(\geq f^{*}(\varphi)\right)} v_{2}(\psi)\right] \\
& =f_{*}\left(\mu_{1}\right)(\varphi)+f_{*}\left(\mu_{2}\right)(\varphi) .
\end{aligned}
$$

Example 2. Let $J: \mathbb{P}^{2} \rightarrow \mathbb{P}^{2}$ be the standard Cremona map given by $J\left[x_{0}: x_{1}: x_{2}\right]=$ $\left[1 / x_{0}: 1 / x_{1}: 1 / x_{2}\right]$. It is a birational map and an involution: $J^{2}=$ the identity map. Let $e_{0}=[1: 0: 0], e_{1}=[0: 1: 0]$ and $e_{2}=[0: 0: 1]$, and $\Sigma_{i}=\left\{x_{i}=0\right\}(i=0,1,2)$. Let $\pi: X \rightarrow \mathbb{P}^{2}$ be the blowup of $\mathbb{P}^{2}$ at $e_{0}, e_{1}$ and $e_{2}$, and let $E_{0}, E_{1}$ and $E_{2}$ be the corresponding exceptional divisors. Let $h=f \circ \pi: X \rightarrow \mathbb{P}^{2}$. Then $h$ is a holomorphic map. Moreover, $\pi^{-1}\left(e_{0}\right)=E_{0}$ and $h\left(E_{0}\right)=\Sigma_{0}$. More precisely, we have $h^{-1}\left(\Sigma_{0} \backslash\left\{e_{1}, e_{2}\right\}\right) \subset E_{0}$. From this, we can compute, as in Example 1 in the introduction and in the proof of part (2) of Proposition 2.5, that for all $\varphi \in C^{0}(X)$ and for $\delta_{e_{0}}$ the Dirac measure at $e_{0}$,

$$
J_{*}\left(\delta_{e_{0}}\right)(\varphi)=\sup _{\Sigma_{0}} \varphi
$$

It follows that $J_{*}\left(\delta_{e_{0}}\right) \geq \max \left\{\delta_{e_{1}}, \delta_{e_{2}}\right\}$, where $\delta_{e_{1}}$ is the Dirac measure at $e_{1}$ and $\delta_{e_{2}}$ is the Dirac measure at $e_{2}$. Therefore, by the positivity of $J_{*}$ we obtain

$$
J_{*} J_{*}\left(\delta_{0}\right)(\varphi) \geq J_{*}\left(\max \left\{\delta_{e_{1}}, \delta_{e_{2}}\right\}\right)(\varphi) \geq \max \left\{J_{*}\left(\delta_{e_{1}}(\varphi)\right), J_{*}\left(\delta_{e_{2}}(\varphi)\right)\right\}=\max \left\{\sup _{\Sigma_{1}} \varphi, \sup _{\Sigma_{2}} \varphi\right\}
$$

On the other hand, $J \circ J$ is the identity map, and hence $(J \circ J)_{*}\left(\delta_{e_{0}}\right)=\delta_{e_{0}}$. Hence the inequality in part (2) of Theorem 2.10 is strict in this case.

This example also shows that the inequality in part (3) of Theorem 2.10 is strict in general. In fact, let $\left\{p_{n}\right\} \subset X \backslash I(f)$ be a sequence converging to a point $p=e_{0}$ and $\left\{J\left(p_{n}\right)\right\}$ converges to a point $q \in \Sigma_{0}$. Let $\mu_{n}=\max \left\{\delta_{p_{1}}, \ldots, \delta_{p_{n}}\right\}$. It can be checked easily that $\mu_{n}$ is an increasing sequence of positive strong submeasures, with $J_{*}\left(\mu_{n}\right)=$ $\max \left\{\delta_{J\left(p_{1}\right)}, \ldots, \delta_{J\left(p_{n}\right)}\right\}$ for all $n$. Then the weak convergence $\operatorname{limit} \mu=\lim _{n \rightarrow \infty} \mu_{n}=$ $\sup _{n} \delta_{p_{n}}$ exists. In particular, $\mu \geq \delta_{e_{0}}$, and hence from the above calculation we find $J_{*}(\mu) \geq \sup _{x \in \Sigma_{0}} \delta_{x}$. On the other hand, $v=\lim _{n \rightarrow \infty} J_{*}\left(\mu_{n}\right)=\sup _{n} \delta_{J\left(p_{n}\right)}$. It is clear that if $x \in \Sigma_{0} \backslash\{q\}$, then $v$ cannot be compared with $\delta_{x}$. Therefore, we have the strict inequality $J_{*}(\mu)>v$ in this case.

If we choose a sequence of points $\left\{p_{n}\right\}_{n=1,2, \ldots} \subset X \backslash\left\{e_{0}, e_{1}, e_{2}\right\}$ converging to $e_{0}$ and such that $q_{n}=J\left(p_{n}\right)$ converges to a point $q_{0} \in \Sigma_{0}$, then it can be seen that for $\mu=$ $\sup _{n} \delta_{p_{n}}$ we have $J_{*}(\mu)>\sup _{n} J_{*}\left(\delta_{p_{n}}\right)$. Hence part (5) of Theorem 2.10 does not hold in general if we replace $\mathcal{G}(\mu)$ by the smaller set $\mathcal{G}=\left\{\delta_{p_{n}}\right\}_{n}$.

2.3. Pullback of positive strong submeasures. Let $f: X \rightarrow Y$ be a continuous open-dense defined map, so that there is an open-dense subset $U \subset X$ such that $f(U)$ is open-dense in $Y$ and $f_{U}=\left.f\right|_{U}: U \rightarrow f(U)$ is a proper covering map of finite degree. In this subsection we will define the pullback of positive strong submeasures on $Y$ for such maps. 
To this end, we note that for each $\varphi \in C^{0}(X)$, the function

$$
\left(\left.f\right|_{U}\right)_{*}(\varphi)(y)=\sum_{x \in\left(\left.f\right|_{U}\right)^{-1}(y)} \varphi(x)
$$

is a continuous function on $f(U)$. Therefore, using Proposition 2.5 we can define the following upper-semicontinuous function on $Y$ :

$$
f_{*}(\varphi):=E\left(\left(\left.f\right|_{U}\right)_{*}(\varphi)\right)
$$

Then, similarly to the previous subsection, we can define for $v \in S M^{+}(Y)$ and $\varphi \in C^{0}(X)$,

$$
f^{*}(v)(\varphi):=\inf _{\psi \in C^{0}\left(Y, \geq f_{*}(\varphi)\right)} v(\psi) .
$$

We have the following relation between pullback and pushforward, whose simple proof is omitted.

THEOREM 2.11. Let $f: X \rightarrow Y$ be a continuous open-dense defined map. Assume that there is an open-dense set $U \subset \operatorname{OpenDom}(f)$ so that $f: U \rightarrow f(U)$ is a homeomorphism. Let $f^{-1}: Y \rightarrow X$ be the inverse open-dense defined map of $f$. Then for all $v \in S M^{+}(Y)$ we have $f^{*}(v)=\left(f^{-1}\right)_{*}(v)$.

The proof of the following result is similar to that of Theorem 2.10.

THEOREM 2.12. Let $X, Y$ be compact metric spaces, and $f: X \rightarrow Y$ a continuous open-dense defined map so that there is an open-dense subset $U \subset X$ such that $f(U)$ is open-dense in $Y$ and $f_{U}=\left.f\right|_{U}: U \rightarrow f(U)$ is a proper covering map of finite degree. Let $g: Y \rightarrow Z$ be another continuous open-dense defined map so that there is an open-dense subset $V \subset Y$ such that $g(V)$ is open-dense in $Y$ and $g_{V}=\left.g\right|_{V}: V \rightarrow g(U)$ is a proper covering map of finite degree.

(1) We have $f^{*}\left(S M^{+}(Y)\right) \subset S M^{+}(X)$. Moreover, if $v \in S M^{+}(Y)$, then $f^{*}(v)( \pm 1)=$ $\operatorname{deg}(f) v( \pm 1)$. In particular, $\left\|f^{*}(v)\right\|=\operatorname{deg}(f)\|v\|$.

(2) For all $v \in S^{+}(Y)$ we have $f^{*} g^{*}(\mu) \geq(g \circ f)^{*}(v)$. If $f$ and $g$ are continuous on the whole of $X$ and $Y$, and $g$ is homeomorphic on an open-dense subset $V$ of $Y$, then equality happens.

\section{Invariant positive strong submeasures}

In the previous section we defined for each continuous open-dense defined map $f: X \rightarrow Y$ an operator $f_{*}: S M^{+}(X) \rightarrow S M^{+}(Y)$. Here we apply this to the case where $X=Y$ to produce invariant positive strong submeasures by combining Cesaro averages with a min-max principle, as stated in Theorem 1.4. We then study further the case where $f$ is good with respect to iterates (Definition 1.5).

We now discuss some properties of invariant positive strong submeasures. We note that in the case where $f$ is a continuous map and $\mu$ is a measure, then $f_{*}(\mu)=\mu$ if and only if either $f_{*}(\mu) \geq \mu$ or $f_{*}(\mu) \leq \mu$. In the general case we are concerned with here, the properties $f_{*}(\mu)=\mu, f_{*}(\mu) \geq \mu$ and $f_{*}(\mu) \leq \mu$ are in general not the same. However, these properties are very much related. For example, it can be checked that if $\mu$ is a measure 
and $f_{*}(\mu) \leq \mu$, then $f_{*}(\mu)=\mu$. On the other hand, we will see that positive strong submeasures $\mu$ having the property that $f_{*}(\mu) \geq \mu$ appear very naturally in dynamics. If we apply Cesaro's average procedure for meromorphic maps, we obtain positive strong submeasures $\mu$ with $f_{*}(\mu) \geq \mu$. Likewise, if we have a positive strong submeasure $\widehat{\mu}$ on $\Gamma_{f, \infty}$ which is $\sigma_{f}$-invariant, then $\mu=\left(\pi_{1}\right)_{*} \widehat{\mu}$ satisfies $f_{*}(\mu) \geq \mu$. Using this, we obtain canonical invariant positive strong submeasures to those $\mu$ which satisfy either $f_{*}(\mu) \geq \mu$ or $f_{*}(\mu) \leq \mu$ in Theorem 1.4. We next detail proofs of these claims.

Proof of Theorem 1.4. (1) We note that for $\mu_{n}=(1 / n) \sum_{j=0}^{n}\left(f_{*}\right)^{j}\left(\mu_{0}\right)$, by part (6) of Theorem 2.10 we have

$$
\begin{aligned}
f_{*}\left(\mu_{n}\right)-\mu_{n} & =f_{*}\left(\frac{1}{n} \sum_{j=0}^{n}\left(f_{*}\right)^{j}\left(\mu_{0}\right)\right)-\frac{1}{n} \sum_{j=0}^{n}\left(f_{*}\right)^{j}\left(\mu_{0}\right) \\
& \geq \frac{1}{n} \sum_{j=0}^{n}\left(f_{*}\right)^{j+1}\left(\mu_{0}\right)-\frac{1}{n} \sum_{j=0}^{n}\left(f_{*}\right)^{j}\left(\mu_{0}\right) \\
& =\frac{1}{n}\left(f_{*}\right)^{n+1}\left(\mu_{0}\right)-\frac{1}{n} \mu_{0},
\end{aligned}
$$

and the latter converges to 0 in $S M(X)$. Therefore, if $\mu=\lim _{j \rightarrow \infty} \mu_{n_{j}}$, then any cluster point of $f_{*}\left(\mu_{n, j}\right)$ is greater than or equal to $\mu$. Hence, by part (3) of Theorem 2.10 we have $f_{*}(\mu) \geq \mu$.

(2) Since $\mu_{n}=\left(f_{*}\right)^{n}\left(\mu_{0}\right)$ is a decreasing sequence, it has a limit which we denote by $\operatorname{Inv}\left(\geq \mu_{0}\right)$, which is an element of $S M^{+}(X)$. Moreover, the sequence $f_{*}\left(\mu_{n}\right)=\mu_{n+1}$ also converges to $\operatorname{Inv}\left(\geq \mu_{0}\right)$. We then have that $f_{*}\left(\operatorname{Inv}\left(\geq \mu_{0}\right)\right) \geq \operatorname{Inv}\left(\geq \mu_{0}\right)$ by part (3) of Theorem 2.10. On the other hand, since $\operatorname{Inv}\left(\geq \mu_{0}\right) \leq \mu_{n}$ for all $n$, it follows that $f_{*} \operatorname{Inv}\left(\geq \mu_{0}\right) \leq f_{*}\left(\mu_{n}\right)$ for all $n$, and hence $f_{*} \operatorname{Inv}\left(\geq \mu_{0}\right) \leq \lim _{n} f_{*}\left(\mu_{n}\right)=\operatorname{Inv}\left(\geq \mu_{0}\right)$. Combining all inequalities, we obtain $f_{*}\left(\operatorname{Inv}\left(\geq \mu_{0}\right)\right)=\operatorname{Inv}\left(\geq \mu_{0}\right)$.

To finish the proof, we will show that if $\mu \leq \mu_{0}$ and $f_{*}(\mu)=\mu$, then $\mu \leq \operatorname{Inv}\left(\geq \mu_{0}\right)$. In fact, under the assumptions about $\mu$ we have

$$
\mu=\left(f_{*}\right)^{n}(\mu) \leq\left(f_{*}\right)^{n}\left(\mu_{0}\right)
$$

for all positive integers $n$. Hence, by taking the limit we obtain that $\mu \leq \operatorname{Inv}\left(\geq \mu_{0}\right)$.

(3) We can assume that the mass of $\mu_{0}$ is 1 . The set $\mathcal{G}:=\left\{\mu \in S M^{+}(X): \mu \geq\right.$ $\left.\mu_{0}, f_{*}(\mu)=\mu\right\}$ is non-empty because $\sup _{x \in X} \delta_{x}$ is one of its elements.

Now let $\mathcal{H}=\left\{v \in M^{+}(X): v \leq \mu, \forall \mu \in \mathcal{G}\right\}$. Note that $\mathcal{H}$ is non-empty because if $v \leq \mu_{0}$, then $v \in \mathcal{H}$. We define $\operatorname{Inv}\left(\geq \mu_{0}\right)=\sup _{v \in \mathcal{H}} v$. Then $\operatorname{Inv}\left(\geq \mu_{0}\right)$ is in $S^{+}(X)$, and it is the largest element of $S M^{+}(X)$ which is smaller than $\mu$ for all $\mu \in \mathcal{G}$. Moreover, by construction we see easily that $\operatorname{Inv}\left(\geq \mu_{0}\right) \geq \mu_{0}$.

We now finish the proof by showing that $f_{*}\left(\operatorname{Inv}\left(\geq \mu_{0}\right)\right)=\operatorname{Inv}\left(\geq \mu_{0}\right)$. First, we show that $\operatorname{Inv}\left(\geq \mu_{0}\right) \geq f_{*}\left(\operatorname{Inv}\left(\geq \mu_{0}\right)\right)$. In fact, it is easy to check that if $v \in M^{+}(X)$ is so that $v \leq \operatorname{Inv}\left(\geq \mu_{0}\right)$, then $v \in \mathcal{H}$. Hence, by part (5) of Theorem 2.10 we have that for all $\mu \in \mathcal{G}$,

$$
f_{*}\left(\operatorname{Inv}\left(\geq \mu_{0}\right)\right)=\sup _{\nu \in \mathcal{H}} f_{*}(\nu) \leq f_{*}(\mu)=\mu .
$$


Therefore, by the definition of $\operatorname{Inv}\left(\geq \mu_{0}\right)$, we get that $f_{*}\left(\operatorname{Inv}\left(\geq \mu_{0}\right)\right) \leq \operatorname{Inv}\left(\geq \mu_{0}\right)$. Therefore, by part (2) above, we have that if $\mu_{\infty}$ is the limit point of $\mu_{n}=\left(f_{*}\right)^{n}(\operatorname{Inv}(\geq$ $\left.\mu_{0}\right)$ ), then $f_{*}\left(\mu_{\infty}\right)=\mu_{\infty}$. Moreover, $\operatorname{Inv}\left(\geq \mu_{0}\right) \geq \mu_{\infty}$.

On the other hand, from the fact that $\operatorname{Inv}\left(\geq \mu_{0}\right) \geq \mu_{0}$ and $f_{*}\left(\mu_{0}\right) \geq \mu_{0}$ we have

$$
\mu_{n}=\left(f_{*}\right)^{n}\left(\operatorname{Inv}\left(\geq \mu_{0}\right)\right) \geq\left(f_{*}\right)^{n}\left(\mu_{0}\right) \geq \mu_{0},
$$

for all $n$. Taking the limit, we obtain $\mu_{\infty} \geq \mu_{0}$, that is, $\mu_{\infty} \in \mathcal{G}$. Hence, by definition $\mu_{\infty} \geq \operatorname{Inv}\left(\geq \mu_{0}\right)$.

From the above two inequalities, we deduce that $\operatorname{Inv}\left(\geq \mu_{0}\right)=\mu_{\infty}$, which implies that $\operatorname{Inv}\left(\geq \mu_{0}\right)$ is the smallest element of $\mathcal{G}$.

Now we consider the case where $f$ is good with respect to iterates; see Definition 1.5. We use the same notation $\Gamma_{f, \infty}, \sigma_{f}: \Gamma_{f, \infty} \rightarrow \Gamma_{f, \infty}$ and $\pi_{1}: \Gamma_{f, \infty} \rightarrow X$ as in the paragraph after Definition 1.5. The next results relate invariant submeasures of $f$ and those of $\sigma_{f}$.

Proposition 3.1. If $\widehat{\mu}$ is a positive strong submeasure on $\Gamma_{f, \infty}$, then

$$
f_{*}\left(\pi_{1}\right)_{*}(\widehat{\mu}) \geq\left(\pi_{1}\right)_{*}\left(\sigma_{f}\right)_{*}(\widehat{\mu}) .
$$

In general, we have that $f_{*}\left(\pi_{1}\right)_{*}(\widehat{\mu}) \neq\left(\pi_{1}\right)_{*}\left(\sigma_{f}\right)_{*}(\widehat{\mu})$, even if $\widehat{\mu}$ is a measure.

Proof of Proposition 3.1. By part (ii) of Theorem 1.2 and the fact that $\sigma_{f}$ and $\pi_{1}$ are continuous maps, we need consider only the case where $\widehat{\mu}$ is a positive measure. We need to show that for all $\varphi \in C^{0}(X)$,

$$
f_{*}\left(\pi_{1}\right)_{*}(\widehat{\mu})(\varphi) \geq\left(\pi_{1}\right)_{*}\left(\sigma_{f}\right)_{*}(\widehat{\mu})(\varphi) .
$$

By definition,

$$
f_{*}\left(\pi_{1}\right)_{*}(\widehat{\mu})(\varphi)=\inf _{\psi \in C^{0}\left(X, \geq f^{*}(\varphi)\right)}\left(\pi_{1}\right)_{*}(\widehat{\mu})(\psi)=\inf _{\psi \in C^{0}\left(X, \geq f^{*}(\varphi)\right)} \widehat{\mu}\left(\pi_{1}^{*}(\psi)\right) .
$$

Let $U=X \backslash I(f)$. Then $U$ is an open-dense subset of $X$ and $\pi_{1}^{-1}(U)$ is an open-dense subset of $\Gamma_{f, \infty}$. Note that $\pi_{1}^{*} \circ f^{*}(\varphi)=\sigma_{f}^{*} \pi_{1}^{*}(\varphi)$ on $\pi_{1}^{-1}(U)$. Moreover, note that the function $\sigma_{f}^{*} \pi_{1}^{*}(\varphi)$ is continuous on the whole $\Gamma_{f, \infty}$. Therefore, for every $\psi \in C^{0}(X, \geq$ $\left.f^{*}(\varphi)\right)$, we have that $\pi_{1}^{*}(\psi) \geq \sigma_{f}^{*} \pi_{1}^{*}(\varphi)$. This implies that

$$
f_{*}\left(\pi_{1}\right)_{*}(\widehat{\mu})(\varphi) \geq \widehat{\mu}\left(\sigma_{f}^{*} \pi_{1}^{*}(\varphi)\right)=\left(\pi_{1}\right)_{*}\left(\sigma_{f}\right)_{*}(\widehat{\mu})(\varphi) .
$$

If we choose an example $f: X \rightarrow X$ having a point $x \in I(f)$ such that $f_{*}\left(\delta_{x}\right)$ is not a measure, then for any $\widehat{x} \in \Gamma_{f, \infty}$ so that $\pi_{1}(\widehat{x})=x$ and $\widehat{\mu}=\delta_{\widehat{x}}$, we have $f_{*}\left(\pi_{1}\right)_{*}(\widehat{\mu}) \neq$ $\left(\pi_{1}\right)_{*}\left(\sigma_{f}\right)_{*}(\widehat{\mu})$, since the left-hand side is not a measure while the right-hand side is a measure.

THEOREM 3.2. Let $f: X \rightarrow X$ be a continuous open-dense defined selfmap of $a$ compact metric space, which is good with respect to iterates. Let $0 \neq \mu_{0} \in S M^{+}(X)$.

(1) Let $\widehat{\mu}$ be a $\sigma_{f}$-invariant positive strong submeasure. Then $\mu=\left(\pi_{1}\right)_{*}(\widehat{\mu})$ satisfies $f_{*}(\mu) \geq \mu$. 
(2) If $f_{*}\left(\mu_{0}\right)=\mu_{0}$, then there exists a non-zero measure $\widehat{\mu_{0}}$ on $\Gamma_{f, \infty}$ so that $\left(\sigma_{f}\right)_{*}\left(\widehat{\mu_{0}}\right)=\widehat{\mu_{0}},\left\|\widehat{\mu_{0}}\right\|=\left\|\mu_{0}\right\|$ and $\left(\pi_{1}\right)_{*}\left(\widehat{\mu_{0}}\right) \leq \mu_{0}$. Moreover, the set $\{\widehat{\mu} \in$ $\left.S M^{+}\left(\Gamma_{f, \infty}\right):\left(\pi_{1}\right)_{*}(\widehat{\mu}) \leq \mu,\left(\sigma_{f}\right)_{*}(\widehat{\mu})=\widehat{\mu}\right\}$ has a largest element, denoted by $\operatorname{Inv}\left(\pi_{1}, \mu\right)$.

Proof. (1) This follows immediately from Proposition 3.1.

(2) Choose any non-zero measure $v_{0}$ on $\Gamma_{f, \infty}$ so that $\left(\pi_{1}\right)_{*}\left(v_{0}\right) \leq \mu_{0}$. Then by Proposition 3.1 we have

$$
\left(\pi_{1}\right)_{*}\left(\sigma_{f}\right)_{*}\left(v_{0}\right) \leq f_{*}\left(\pi_{1}\right)_{*}\left(v_{0}\right) \leq f_{*}\left(\mu_{0}\right)=\mu_{0} .
$$

Hence any cluster point $v$ of Cesaro's averages $(1 / n) \sum_{j=0}^{n}\left(\sigma_{f}\right)_{*}\left(v_{0}\right)$ will satisfy $\left(\pi_{1}\right)_{*}(v) \leq \mu$. Now to construct one that has the same mass as $\mu$, we choose a sequence of positive measures $\mu_{n}$ with the property $\mu_{n} \leq \mu$ and $\left\|\mu_{n}\right\| \rightarrow\|\mu\|$. For each $\mu_{n}$, construct a corresponding $v_{n}$ on $\Gamma_{f, \infty}$ as above. Then any cluster point $\widehat{\mu_{0}}$ of $v_{n}$ will satisfy the properties in line 1 of part 2 of the statement of Theorem 3.2.

Since $\sigma_{f}$ is continuous, it follows that $\left(\sigma_{f}\right)_{*}(v)=v$. If we $\operatorname{define} \operatorname{Inv}\left(\pi_{1}, \mu\right)=$ $\sup \left\{\widehat{\mu} \in S M^{+}\left(\Gamma_{f, \infty}\right):\left(\pi_{1}\right)_{*}(\widehat{\mu}) \leq \mu, \quad\left(\sigma_{f}\right)_{*}(\widehat{\mu})=\widehat{\mu}\right\}$, then it is also an element of $S M^{+}\left(\Gamma_{f, \infty}\right)$. Moreover, since $\sigma_{f}$ is continuous, we can check easily that $\operatorname{Inv}\left(\pi_{1}, \mu\right)$ is $\sigma_{f}$-invariant.

In summary, we have several canonical ways to associate invariant positive strong submeasures on $X$ or $\Gamma_{f, \infty}$. If $\widehat{\mu}$ is an invariant positive strong submeasure on $\Gamma_{f, \infty}$, then we obtain an invariant positive strong submeasure $\operatorname{Inv}\left(\geq\left(\pi_{1}\right)_{*}(\widehat{\mu})\right)$ on $X$. Conversely, if $\mu$ is an invariant positive strong submeasure on $X$, then we obtain an invariant positive strong submeasure $\operatorname{Inv}\left(\pi_{1}, \mu\right)$ on $\Gamma_{f, \infty}$. If $\mu$ is any positive strong submeasure on $X$ and $\mu_{\infty}$ is any cluster point of Cesaro's average $(1 / n) \sum_{j=0}^{n}\left(f_{*}\right)^{j}\left(\mu_{\infty}\right)$, then we obtain an invariant positive strong submeasure $\operatorname{Inv}\left(\geq \mu_{\infty}\right)$.

\section{Applications}

In this section we provide some applications of the previous sections. Firstly, we will consider a general dominant meromorphic selfmap. Then we consider in more detail dominant meromorphic selfmaps in dimension 2. Finally, we consider the case of transcendental maps of $\mathbb{C}$ and $\mathbb{C}^{2}$.

4.1. Dominant meromorphic maps. Let $X$ be a compact complex variety and $f$ : $X \rightarrow X$ a dominant meromorphic map. Then, from the topological viewpoint, $f$ is an open-dense defined selfmap which is good with respect to iterates. Therefore, we can define the pushforward of a positive strong submeasure by $f$, as well as the notions of topological entropy.

We can also define the pullback of positive strong submeasures by $f$, following the description in $\$ 2.3$. We end this section describing in detail the pushforward by meromorphic maps on positive strong submeasures. The next result about a good choice of $\psi \in C^{0}(X, \geq \varphi)$ for some special bounded upper-semicontinuous functions will be needed for that purpose. 
Lemma 4.1. Let $X$ be a compact metric space, $A \subset X$ a closed set and $U=X \backslash A$. Let $\varphi$ be a bounded upper-semicontinuous function on $X$ so that $\chi=\left.\varphi\right|_{U}$ is continuous on $U$ and $\gamma=\left.\varphi\right|_{A}$ is continuous on A. For any $U^{\prime} \subset \subset U$ an open set and $\epsilon>0$, there is a function $\psi \in C^{0}(X, \geq \varphi)$ so that:

(i) $\left.\psi\right|_{U^{\prime}}=\chi$;

(ii) $\sup _{A}|\psi|_{A}-\gamma \mid \leq \epsilon$; and

(iii) $\sup _{X}|\psi| \leq \sup _{X}|\varphi|+\epsilon$.

Proof. Let $\epsilon_{1}>0$ be a small number to be determined later. Since $\varphi$ is uppersemicontinuous, for each $x \in A$, there is $r_{x}>0$, which we choose so small that $\overline{U^{\prime}} \cap \overline{B\left(x, r_{x}\right)}=\emptyset$, so that

$$
\sup _{y \in U \cap \overline{B\left(x, r_{x}\right)}} \chi(y) \leq \gamma(x)+\epsilon_{1} .
$$

Since $\gamma$ is continuous on $A$, by shrinking $r_{x}$ if necessary, we can assume that

$$
\sup _{x^{\prime} \in A \cap \overline{B\left(x, r_{x}\right)}}\left|\gamma\left(x^{\prime}\right)-\gamma(x)\right| \leq \epsilon_{1} .
$$

Hence we obtain

$$
\sup _{y \in U \cap \overline{B\left(x, r_{x}\right)}} \chi(y) \leq \inf _{x^{\prime} \in A \cap \overline{B\left(x, r_{x}\right)}} \gamma\left(x^{\prime}\right)+2 \epsilon_{1} .
$$

The function $\left.\gamma\right|_{A \cap \overline{B\left(x, r_{x}\right)}}$ can be extended to a continuous function $\gamma_{x}$ on $\overline{B\left(x, r_{x}\right)}$. We can assume, by shrinking $r_{x}$ for example, that

$$
\sup _{x^{\prime}, x^{\prime \prime} \in \overline{B\left(x, r_{x}\right)}}\left|\gamma_{x}\left(x^{\prime}\right)-\gamma_{x}\left(x^{\prime \prime}\right)\right| \leq \epsilon_{1} .
$$

Now, since $A$ is compact, we can find a finite number of such balls, say $B\left(x_{1}, r_{1}\right), \ldots$, $B\left(x_{m}, r_{m}\right)$, which cover $A$. We choose another open subset $U^{\prime \prime}$ of $X$ so that $U^{\prime} \subset \subset$ $U^{\prime \prime} \subset \subset U$ and so that $U^{\prime \prime}, B\left(x_{1}, r_{1}\right), \ldots, B\left(x_{m}, r_{m}\right)$ is a finite open covering of $X$. Let $\tau, \tau_{1}, \ldots, \tau_{m}$, be a partition of unity subordinate to this open covering. Then the function

$$
\psi(x)=\tau(x) \gamma(x)+\sum_{i=1}^{m} \tau_{i}(x)\left[\gamma_{x_{i}}(x)+4 \epsilon_{1}\right],
$$

with $4 \epsilon_{1}<\epsilon$, satisfies the conclusion of the lemma.

Proof of Theorem 1.3. Let $B \in Z$ be the exceptional divisor of the blowup. Then $\pi$ : $B \rightarrow A$ is a smooth holomorphic fibration, whose fibres are isomorphic to $\mathbb{P}^{r-1}$ where $r$ is the codimension of $A$. As in Example 1, it can be computed for $x \in A$ that $\pi_{*}(\varphi)(x)=$ $\sup _{y \in \pi^{-1}(x)} \varphi$. Therefore, from what was said about the map $\pi: B \rightarrow A$, it follows that $\left.\pi_{*}(\varphi)\right|_{A}$ is continuous. Then it is easy to see that the upper-semicontinuous function $\pi_{*}(\varphi)$ satisfies the conditions of Lemma 4.1. It is easy to check that $\pi^{*}\left(\mu_{1}\right)$ is a positive measure on $Z$. Hence, by the conclusion of Lemma 4.1 , it is easy to see that

$$
\pi^{*}(\mu)(\varphi)=\pi^{*}\left(\mu_{1}\right)(\varphi)+\mu_{2}\left(\left.\pi_{*}(\varphi)\right|_{A}\right) .
$$


Now we provide an explicit choice of the collection $\mathcal{G}$ associated to $\pi^{*}(\mu)$ in part (2) of Theorem 2.1. From equation (4.1), it suffices to provide such a $\mathcal{G}$ for $\mu_{2}$, since then the corresponding collection for $\mu$ will be $\pi^{*} \mu_{1}+\mathcal{G}$. Therefore, in the remainder of the proof, we will assume that $\mu=\mu_{2}$ has support on $A$.

Define $\psi(\varphi)=\left.\pi_{*}(\varphi)\right|_{A}$. It then follows that $\psi(\varphi) \in C^{0}(A)$, and

$$
\pi^{*}(\mu)(\varphi)=\mu(\psi(\varphi))
$$

We now present an explicit collection $\mathcal{G}$ of positive measures on $X$ so that

$$
\mu(\psi(\varphi))=\sup _{\chi \in \mathcal{G}} \chi(\varphi) .
$$

To this end, let us, for each finite open cover $\left\{U_{i}\right\}_{i \in I}$ of $A$, a partition of unity $\left\{\tau_{i}\right\}$ subordinate to the finite open cover $\left\{U_{i}\right\}$ of $A$, and local continuous sections $\gamma_{i}: U_{i} \rightarrow$ $\pi^{-1}\left(U_{i}\right)$, consider the following assignment on $B$ :

$$
\chi\left(\left\{U_{i}\right\},\left\{\tau_{i}\right\}, \gamma_{i}\right)(\varphi)=\mu(H(\varphi)),
$$

where $H(\varphi) \in C^{0}(A)$ is the function

$$
H(\varphi)(x)=\sum_{i \in I} \tau_{i}(x) \varphi\left(\gamma_{i}(x)\right)
$$

Since $H(\varphi)$ is linear and non-decreasing in $\varphi$, it is easy to see that $\chi$ is indeed a measure. Moreover, since $|H(x)| \leq \max _{B}|\varphi|$, it follows that $\|\chi\| \leq\|\mu\|$.

We let $\mathcal{G}$ be the collection of such positive measures. We now claim that, for all $\varphi \in$ $C^{0}(Z)$,

$$
\mu(\psi(\varphi))=\sup _{\chi \in \mathcal{G}} \chi(\varphi)
$$

We show first that $\mu(\psi(\varphi)) \geq \chi(\varphi)$ for all $\chi \in \mathcal{G}$. In fact, since $\gamma_{i}(x) \in \pi^{-1}(x)$ for all $x \in A$, it follows by definition that

$$
H(\varphi)(x) \leq \sup _{\pi^{-1}(x)} \varphi=\psi(\varphi)(x),
$$

for all $x \in A$. Hence $\mu(\psi(\varphi)) \geq \chi(\varphi)$.

Now we show the converse. Let $\varphi$ be any continuous function on $Z$. Then for any $\epsilon>0$ we can always find a finite open covering $\left\{U_{i}\right\}_{i \in I}$ of $X$, depending on $\varphi$ and $\epsilon$, so that for all $x \in U_{i}$ we have

$$
\left|\varphi\left(\gamma_{i}(x)\right)-\sup _{\pi^{-1}(x)} \varphi\right| \leq \epsilon .
$$

It then follows that correspondingly $|H(x)-\psi(\varphi)(x)| \leq \epsilon$ for all $x \in A$. Therefore, for this choice of $\chi \in \mathcal{G}$,

$$
|\mu(\psi(\varphi))-\mu(H)| \leq \epsilon,
$$

and hence letting $\epsilon \rightarrow 0$ concludes the proof. 
4.2. Meromorphic maps in dimension 2. In this subsection we mention an application to dynamics of meromorphic maps. Let $f: X \rightarrow X$ be a dominant meromorphic map of a compact Kähler surface. The study of dynamics of such maps is very active. It is now recognized that maps which are algebraic stable (those whose pullback on cohomology groups is compatible with iterates, that is, $\left(f^{n}\right)^{*}=\left(f^{*}\right)^{n}$ on $H^{1,1}(X)$ for all $\left.n \geq 0\right)$ have good dynamical properties. An important indication of the complexity of such maps is dynamical degrees defined as follows. Let $\lambda_{1}(f)$ be the spectral radius of the linear map $f^{*}: H^{1,1}(X) \rightarrow H^{1,1}(X)$ and let $\lambda_{2}(f)$ be the spectral radius of the linear map $f^{*}: H^{2,2}(X) \rightarrow H^{2,2}(X)$. There are two interesting large classes of such maps: those with large topological degree $\left(\lambda_{2}(f)>\lambda_{1}(f)\right)$ and those with large first dynamical degree $\left(\lambda_{1}(f)>\lambda_{2}(f)\right)$. The dynamics of the first class is shown in our paper [9] to be as nice as expected. For the second class, the most general result so far belongs to [5-7], who showed the existence of canonical Green $(1,1)$ currents $T^{+}$and $T^{-}$for $f$, and who used potential theory to prove that the dynamics is nice (in particular, the wedge intersection $T^{+} \wedge T^{-}$ is well defined as a positive measure) if the so-called finite energy conditions on the Green currents are satisfied. While these conditions are satisfied for many interesting subclasses, it is known that in general they are false [4]. On the other hand, since it is known that $T^{+}$has no mass on curves [7], it follows that the least negative intersection $\Lambda\left(T^{+}, T^{-}\right)$ defined in [20] is a positive strong submeasure (see the proof therein). In summary, we obtain the following result.vspace-1.5pt

THEOREM 4.2. Let $f: X \rightarrow X$ be a dominant meromorphic map of a compact Kähler surface which is algebraic stable and has $\lambda_{1}(f)>\lambda_{2}(f)$. Let $T^{+}$and $T^{-}$be the canonical Green $(1,1)$ currents of $f$. Then the least negative intersection $\Lambda\left(T^{+}, T^{-}\right)$is in $S M^{+}(X)$.

At the moment we do not know whether $f_{*}\left(\Lambda\left(T^{+}, T^{-}\right)\right)=\Lambda\left(T^{+}, T^{-}\right)$. (In fact, from Buff's examples mentioned above, we do not expect this to be true in general.) However, by the discussion in the previous sections, we can consider cluster points of Cesaro's average $(1 / n) \sum_{j=1}^{n}\left(f_{*}\right)^{j}\left(\Lambda\left(T^{+}, T^{-}\right)\right)$, and then obtain the associated invariant positive strong submeasures $\mu$. From such a $\mu$, we then can construct associated invariant measures of $\sigma_{f}$. The significance of these invariant positive strong submeasures and measures, together with their entropies, will be pursued in a future work. (The cluster points of Cesaro's average can be worked out explicitly when we work with a transcendental holomorphic map of $\mathbb{C}^{1}$ and start with a probability measure $\mu$ on $\mathbb{P}^{1}$. In this case, thanks to Picard's theorem for essential singularities, the only dynamically interesting invariant positive strong submeasure we can obtain is $\sup _{x \in \mathbb{P}^{1}} \delta_{x}$. The computations are left to the interested reader to carry out.)

Remark. We note a parallel between the least negative intersection and the tangent currents in this situation. Under the same assumptions as in Theorem 4.2, it was shown in our joint paper [8] that the h-dimension (defined in [10]) between $T^{+}$and $T^{-}$is 0 , the best possible.

Remark. In this paper we only treat existence of invariant strong submeasure for open-dense defined maps. Applications to finer invariants such as topological entropy will 
be pursued in a future paper. For related classical works, see [1, 13]. For some recent related works, see $[3,21]$.

Acknowledgments. This paper is extracted and developed from the more dynamical part of our preprint [20]. The part about the least negative intersection of positive closed $(1,1)$ currents will be separated into another paper. We thank the anonymous referees for helpful feedback. The author is supported by Young Research Talents grant 300814 from the Research Council of Norway.

\section{REFERENCES}

[1] R. L. Adler, A. G. Konheim and M. H. McAndrew. Topological entropy. Trans. Amer. Math. Soc. 114 (1965), 309-319.

[2] R. Baire. Leçons sur les fonctions discontinues, professées au collège de France. Gauthier-Villars, Paris, 1905.

[3] A. M. Benini, J. E. Fornæss and H. Peters. Entropy of transcendental entire functions. Ergod. Th. \& Dynam. Sys. 41(2) (2020), 338-348.

[4] X. Buff. Courants dynamiques pluripolaires. Ann. Fac. Sci. Toulouse Math. 20(1) (2011), 203-214.

[5] J. Diller, R. Dujardin and V. Guedj. Dynamics of meromorphic maps with small topological degrees III: Geometric currents and ergodic theory. Ann. Sci. Éc. Norm. Supér. 43 (2010), 235-378.

[6] J. Diller, R. Dujardin and V. Guedj. Dynamics of meromorphic maps with small topological degrees II: Energy and invariant measures. Comment. Math. Helv. 86 (2011), 277-316.

[7] J. Diller, R. Dujardin and V. Guedj. Dynamics of meromorphic maps with small topological degrees I: From currents to cohomology. Indiana Univ. Math. J. 59 (2010), 521-561.

[8] T.-C. Dinh, V.-A. Nguyen and T. T. Truong. Growth of the number of periodic points of meromorphic maps. Bull. Lond. Math. Soc. 49(6) (2017), 947-964.

[9] T.-C. Dinh, V.-A. Nguyen and T. T. Truong. Equidistribution for meromorphic maps with dominant topological degree. Indiana Univ. Math. J. 64(6) (2015), 1805-1828.

[10] T.-C. Dinh and N. Sibony. Density of positive closed currents, a theory of non-generic intersection. J. Algebraic Geom., to appear. arXiv:1203.5810.

[11] T.-C. Dinh and N. Sibony. Regularization of currents and entropy. Ann. Sci. Éc. Norm. Supér. 37(6) (2004), 959-971.

[12] J. L. Doob. Measure Theory (Graduate Text in Mathematics, 143). Springer, New York, 1994.

[13] P. Fatou. Sur l'iteration des fonctions transcendantes entires. Acta Math. 47(4) (1926), 337-370.

[14] T. N. T. Goodman. Relating topological entropy and measure entropy. Bull. Lond. Math. Soc. 3 (1971), 176-180.

[15] L. W. Goodwyn. Comparing topological entropy with measure-theoretic entropy. Amer. J. Math. 94(2) (1972), 366-388.

[16] M. Méo. Inverse image of a closed positive current by a surjective analytic map. C. R. Math. Acad. Sci. Paris 322(2) (1996), 1141-1144 (in French).

[17] W. Rudin. Functional Analysis, 2nd edn. McGraw-Hill, New York, 1991.

[18] W. Rudin. Real and Complex Analysis, 3rd edn. McGraw-Hill, New York, 1987.

[19] M. Talagrand. Maharam's problem. Ann. of Math. 168 (2008), 981-1009.

[20] T. T. Truong. Submeasures and several applications. Preprint, 2019, arXiv: 1712.02490.

[21] T. T. Truong. Etale dynamical systems and topological entropy. Proc. Amer. Math. Soc., to appear. Preprint, 2016, arXiv:1607.07412. 